\title{
GROMOV-WITTEN THEORY AND CYCLE-VALUED MODULAR FORMS
}

\author{
TODOR MILANOV \& YONGBIN RUAN \& YEFENG SHEN
}

\section{Contents}

1. Introduction

2. Cohomological field theory and quantization

3. Global Frobenius manifolds for simple elliptic singularities

4. Global B-model CohFT and anti-holomorphic completion

5. A-model CohFT and cycle valued modular forms

References

\section{INTRODUCTION}

A remarkable phenomenon in Gromov-Witten theory is the appearance of (quasi) modular forms. Classically, modular forms arise as a counting function of points, i.e., zero dimensional objects. A Gromov-Witten generating function can be thought as a counting function for the virtual number of holomorphic curves, i.e., one dimensional objects. Therefore, it is natural to speculate if modular forms appear here too. One can attempt to compute them explicitly. If one is lucky enough, the answers can be organized as modular forms. Indeed, this strategy has been carried out for elliptic curves OP and the so called reduced Gromov-Witten theory of K3-surfaces [MPT]. However, we should emphasize that both steps of the strategy are highly nontrivial. In fact, the above modularity results are some of the most sophisticated works in GromovWitten theory. Generally speaking, it is very difficult to compute GromovWitten invariants. Even if you can compute, it is not clear how to organize them into modular forms. Unlike the case of counting points, it is impractical to try to compute a large number of coefficients and then guess the general pattern.

In the middle of the 90's, by studying the physical B-model of GromovWitten theory, BCOV boldly conjectured that Gromov-Witten generating function of any Calabi-Yau manifolds are in fact quasi-modular forms. A key idea in [BCOV] is that the B-model Gromov-Witten function should be 
modular but non-holomorphic. Furthermore, its anti-holomorphic dependence is governed by the famous holomorphic anomaly equations. During the last decade, Klemm and his collaborators have put forth a series of papers to solve the holomorphic anomaly equations [ABK, HKQ]. One upshot is a stunning predication of Gromov-Witten invariants of quintic 3-fold up to genus 51 . Indeed, this is a great achievement since mathematicians can only compute Gromov-Witten invariants for genus zero and one. Motivated by the physical intuition, there were two independent works recently in mathematics to establish the modularity of Gromov-Witten theory rigorously for local $\mathbb{P}^{2}$ CI2 and elliptic orbifolds $\mathbb{P}^{1}[\mathrm{KS}, \mathrm{MR}$. Let's briefly describe the authors' work on the elliptic orbifolds $\mathbb{P}^{1}$. The current article can be thought as a sequel.

Let $X$ be a projective manifold and $\overline{\mathcal{M}}_{g, n}(X, \beta)$ be the moduli space of genus- $g$, degree- $\beta$ stable maps with $n$ markings, where $\beta$ is a nef class in $H^{2}(X, \mathbb{Z})$, i.e., $\beta \in \mathrm{NE}(X)$. Let $\mathrm{ev}_{i}$ be the evaluation map at the $i$-th marked point $p_{i}$ and $\psi_{i} \in H^{*}\left(\overline{\mathcal{M}}_{g, n}\right)$ be the first Chern class of the cotangent line bundle at $p_{i}$. Choose elements $\gamma_{i}$ in $H^{*}(X, \mathbb{Q})$ with $\gamma_{0}=1 \in H^{0}(X, \mathbb{Q})$. $\pi: \overline{\mathcal{M}}_{g, n}(X, \beta) \rightarrow \overline{\mathcal{M}}_{g, n}$ be the stabilization of the forgetful morphism. The numerical GW invariants with ancestors are defined by

$$
\left\langle\tau_{\iota 1}\left(\gamma_{1}\right), \cdots, \tau_{\iota_{n}}\left(\gamma_{n}\right)\right\rangle_{g, n, \beta}^{X}=\int_{\left[\overline{\mathcal{M}}_{g, n}(X, \beta)\right]^{\mathrm{vir}}} \prod_{i=1}^{n} \operatorname{ev}_{i}^{*}\left(\gamma_{i}\right) \cup \pi^{*} \psi_{i}^{\iota_{i}} .
$$

The above invariant is zero unless

$$
\sum_{i=1}^{n}\left(\operatorname{deg}_{\mathbb{C}}\left(\gamma_{i}\right)+\iota_{i}\right)=c_{1}(T X) \cdot \beta+\left(3-\operatorname{dim}_{\mathbb{C}} X\right)(g-1)+n .
$$

The advantage of Calabi-Yau manifolds, such as the elliptic curve $E$, is that $c_{1}(T X)=0$ and hence the dimension constraint is independent of $\beta$. For the elliptic curve $E$, the degree $\beta=d \cdot \mathcal{D}$, where $d$ is a non-negative integer and $\mathcal{D}$ is a nef generator of $H^{2}(E, \mathbb{Z})$. Then, it is natural to define

$$
\left\langle\tau_{\iota_{1}}\left(\gamma_{1}\right), \cdots, \tau_{\iota_{n}}\left(\gamma_{n}\right)\right\rangle_{g, n}^{E}(q)=\sum_{d \geq 0}\left\langle\tau_{\iota_{1}}\left(\gamma_{1}\right), \cdots, \tau_{\iota_{n}}\left(\gamma_{n}\right)\right\rangle_{g, n, d}^{E} q^{d}
$$

where $q$ is the Novikov variable that we use to keep track of the degree $\beta$. In our case, the function (1.2) can be seen as an ancestor Gromov-Witten function along $t \cdot \mathcal{D} \in H^{2}(X, \mathbb{Z})$ by setting $q=e^{t}$ (see Section 5 ). The authors proved the modularity for the elliptic orbifolds $\mathbb{P}^{1}$ with weights of non-trivial orbifold points are $(3,3,3),(2,4,4),(2,3,6)$. These orbifolds are the quotients of some elliptic curve $E$ by $\mathbb{Z} / 3 \mathbb{Z}, \mathbb{Z} / 4 \mathbb{Z}, \mathbb{Z} / 6 \mathbb{Z}$ respectively.

To state the theorem, let $\mathcal{X}$ be one of the three elliptic orbifolds $\mathbb{P}^{1}$. Again, $c_{1}(T \mathcal{X})=0$ in these cases. We can choose elements $\gamma_{i}$ of $H_{C R}^{*}(\mathcal{X})$ and define

$$
\left\langle\tau_{\iota_{1}}\left(\gamma_{i_{1}}\right), \cdots, \tau_{\iota_{n}}\left(\gamma_{i_{n}}\right)\right\rangle_{g, n}^{\mathcal{X}}(q)
$$


similarly. The main result of [KS, $[\mathrm{MR}]$ is the following modularity theorem.

Theorem 1.1. $\mathrm{MR}$ Suppose that $\mathcal{X}$ is one of the three elliptic orbifolds $\mathbb{P}^{1}$ from above. For any multi-indices $\iota_{j}, i_{j}$, the $G W$ invariant (1.3) converges to a quasi-modular form of an appropriate weight for a finite index subgroup $\Gamma$ of $S L_{2}(\mathbb{Z})$ under the change of variables $q=e^{2 \pi i \tau / 3}, e^{2 \pi i \tau / 4}, e^{2 \pi i \tau / 6}$, respectively (see [MR] for the subgroup $\Gamma$ and the weights of the quasi-modular forms).

The same theorem for elliptic curves were proved ten years ago by OkounkovPandharipande $\mathrm{OP}$.

Recall that one can construct Gromov-Witten cycles (cohomological field theories) by a partial integration, i.e., pushforward via the forgetfull morphism

$$
\Lambda_{g, n, \beta}^{X}\left(\gamma_{1}, \cdots, \gamma_{n}\right)=\pi_{*}\left(\prod_{i=1}^{n} \operatorname{ev}_{i}^{*}\left(\gamma_{i}\right)\right) \in H^{*}\left(\overline{\mathcal{M}}_{g, n}, \mathbb{Q}\right) .
$$

The degree of the cycle is computed from the dimension axiom,

$$
\operatorname{deg}_{\mathbb{C}} \Lambda_{g, n, \beta}^{X}\left(\gamma_{1}, \cdots, \gamma_{n}\right)=(g-1) \operatorname{dim}_{C}(X)+\sum_{i=1}^{n} \operatorname{deg}_{\mathbb{C}}\left(\gamma_{i}\right)-c_{1}(T X) \cdot \beta .
$$

The numerical Gromov-Witten invariants are obtained by

$$
\left\langle\tau_{\iota_{1}}\left(\gamma_{1}\right), \cdots, \tau_{\iota_{c}}\left(\gamma_{n}\right)\right\rangle_{g, n, \beta}^{X}=\int_{\overline{\mathcal{M}}_{g, n}} \Lambda_{g, n, \beta}^{X}\left(\gamma_{1}, \cdots, \gamma_{n}\right) \cup \prod_{i=1}^{n} \psi_{i}^{\iota_{i}} .
$$

Motivated by the corresponding work in number theory [Z], we want to consider the generating function of Gromov-Witten cycles

$$
\Lambda_{g, n}^{X}\left(\gamma_{1}, \cdots, \gamma_{n}\right)(q)=\sum_{\beta \in \operatorname{NE}(X)} \Lambda_{g, n, \beta}^{X}\left(\gamma_{1}, \cdots, \gamma_{n}\right) q^{\beta} .
$$

We view the RHS of (1.5) as a function on $q$ taking value in $H^{*}\left(\overline{\mathcal{M}}_{g, n}, \mathbb{Q}\right)$. To emphasise this perspective, we sometimes refer to it as cycle-valued generating function. The main theorem of this paper is

Theorem 1.2. Suppose that $\mathcal{X}$ is one of the three elliptic orbifolds $\mathbb{P}^{1}$ with three non-trivial orbifold points; then $\Lambda_{g, n}^{\mathcal{X}}\left(\gamma_{1}, \cdots, \gamma_{n}\right)(q)$ converges to a cyclevalued quasi-modular form of an appropriate weight for a finite index subgroup $\Gamma$ of $S L_{2}(\mathbb{Z})$ under the change of variables $q=e^{2 \pi i \tau / 3}, e^{2 \pi i \tau / 4}, e^{2 \pi i \tau / 6}$, respectively.

We should mention that the above cycle-valued modularity theorem is not yet known for elliptic curve.

We obtain the modularity of numerical Gromov-Witten invariants by integrating the $\Lambda_{g, n}^{\mathcal{X}}\left(\gamma_{1}, \cdots, \gamma_{n}\right)$ with psi-classes over the fundamental cycle $\left[\overline{\mathcal{M}}_{g, n}\right]$. 
On the other hand, we can also use other interesting classes of $\overline{\mathcal{M}}_{g, n}$ such as $\kappa_{i}$ 's or Hodge class $\lambda_{i}$ 's.

Suppose that $P$ is a polynomial of $\psi_{i}, \kappa_{i}, \lambda_{i}$. We define a generalized numerical Gromov-Witten invariants

$$
\left\langle\gamma_{1}, \cdots, \gamma_{n} ; P\right\rangle_{g, n, \beta}^{X}=\int_{\overline{\mathcal{M}}_{g, n}} P \cup \Lambda_{g, n, \beta}^{X}\left(\gamma_{1}, \cdots, \gamma_{n}\right)
$$

and its generating function

$$
\left\langle\gamma_{1}, \cdots, \gamma_{n} ; P\right\rangle_{g, n}^{X}(q)=\sum_{\beta \in \operatorname{NE}(X)}\left\langle\gamma_{1}, \cdots, \gamma_{n} ; P\right\rangle_{g, n, \beta}^{X} q^{\beta} .
$$

Here, we set it to be zero if the dimension constraint are not satisfied.

Corollary 1.3. Suppose that $\mathcal{X}$ is one of the above three elliptic orbifolds $\mathbb{P}^{1}$. Then, the above generalized numerical Gromov-Witten generating functions are quasi-modular forms for the same modular group and weights given by the main theorem.

Recall that the proof of the numerical version consists of two steps. The first step is to construct a higher genus B-model theory (modulo an extension problem) and prove its modularity. Then, the second step is to prove mirror theorems to match it with a Gromov-Witten theory which will solve the extension property as well as inducing the modularity for a Gromov-Witten theory. In this paper, we follow the same outline, i.e., our strategy can be carried out on the cycle level. Our main new ingredient is Teleman's reconstruction theorem [T].

The paper is organized as follows. In Section 2, we will review the action of upper-triangular symplectic operators on a cohomological field theory, which will be the main tool of the paper. In Section 3, we review the construction of global Frobenius manifold structures from [MR. Using it, we can define Givental B-model cohomological field theory as indicated by Telemann [T]. In Section 4, we calculate the action of the monodromy group on the Givental's B-model cohomological field theory and prove the (quasi-)modularity. Finally, in Section 5, we prove the mirror theorems on the cycle level. Here, the original $g$-reduction argument does not apply. We replace it by Teleman's reconstruction theorem $[\mathrm{T}]$.

1.1. Acknowledgements. The work of the first author is supported by GrantIn-Aid and by the World Premier International Research Center Initiative (WPI Initiative), MEXT, Japan. The second author is partially supported by a NSF grant. The second and third authors would like to thank Hiroshi Iritani for interesting discussions on the convergence of Gromov-Witten theory. The third author would like to thank Emily Clader, Nathan Priddis and 
Mark Shoemaker for helpful discussions on Givental's theory. Finally, three of us would like to thank IPMU for hospitality where the part of this work is carried out. We thank Arthur Greenspoon for editorial assistance.

\section{Cohomological Field Theory AND QUANTization}

The quantization formalism in Gromov-Witten theory was introduced by Givental in G1 and then revisited by Teleman at the cohomological field theory level in [T]. The latter will be used in this article. For the readers'

convenience, we give a brief introduction here. $\pi_{g, n, k}: \overline{\mathcal{M}}_{g, n+k} \rightarrow \overline{\mathcal{M}}_{g, n}$ be the stabilization of the morphism that forgets the last $k$ marked points. For simplicity, we will omit the subscripts if they are indicated in the context.

2.1. Cohomological field theories. Let $H$ be a vector space of dimension $N$ with a unit 1 and a non-degenerate paring $\eta$. Without loss of generality, we always fix a basis of $H$, say $\mathscr{S}:=\left\{\partial_{i}, i=0, \cdots, N-1\right\}$, and we set $\partial_{0}=1$. Let $\left\{\partial^{j}\right\}$ be the dual basis in the dual space $H^{\vee}$, (i.e., $\left.\eta\left(\partial_{i}, \partial^{j}\right)=\delta_{i}^{j}\right)$. A Cohomological field theory (or CohFT) is a set of multi-linear maps $\Lambda=\left\{\Lambda_{g, n}\right\}$, with

$$
\Lambda_{g, n}: H^{\otimes n} \longrightarrow H^{*}\left(\overline{\mathcal{M}}_{g, n}, \mathbb{C}\right)
$$

or equivalently,

$$
\Lambda_{g, n} \in H^{*}\left(\overline{\mathcal{M}}_{g, n}, \mathbb{C}\right) \otimes\left(H^{\vee}\right)^{\otimes n}
$$

defined for each stable genus $g$ curve with $n$ marked points,i.e., $2 g-2+n>0$. Furthermore, $\Lambda$ satisfies a set of axioms (CohFT axioms) described below:

(i) $\left(S_{n}\right.$-invariance) For any $\sigma \in S_{n}$, and $\gamma_{1}, \cdots, \gamma_{n} \in H$; then

$$
\Lambda_{g, n}\left(\gamma_{\sigma(1)}, \cdots, \gamma_{\sigma(n)}\right)=\Lambda_{g, n}\left(\gamma_{1}, \cdots, \gamma_{n}\right) \text {. }
$$

(ii) (Gluing tree) Let

$$
\rho_{\text {tree }}: \overline{\mathcal{M}}_{g_{1}, n_{1}+1} \times \overline{\mathcal{M}}_{g_{2}, n_{2}+1} \rightarrow \overline{\mathcal{M}}_{g, n}
$$

where $g=g_{1}+g_{2}, n=n_{1}+n_{2}$, be the morphism induced from gluing the last marked point of the first curve and the first marked point of the second curve; then

$$
\begin{aligned}
\rho_{\text {tree }}^{*} & \left(\Lambda_{g, n}\left(\gamma_{1}, \cdots, \gamma_{n}\right)\right) \\
& =\sum_{\alpha, \beta \in \mathscr{S}} \Lambda_{g_{1}, n_{1}+1}\left(\gamma_{1}, \cdots, \gamma_{n_{1}}, \alpha\right) \eta^{\alpha, \beta} \Lambda_{g_{2}, n_{2}+1}\left(\beta, \gamma_{n_{1}+1}, \cdots, \gamma_{n}\right) .
\end{aligned}
$$

Here $\left(\eta^{\alpha, \beta}\right)_{N \times N}$ is the inverse matrix of $(\eta(\alpha, \beta))_{N \times N}$.

(iii) (Gluing loop) Let

$$
\rho_{\text {loop }}: \overline{\mathcal{M}}_{g-1, n+2} \rightarrow \overline{\mathcal{M}}_{g, n},
$$


be the morphism induced from gluing the last two marked points; then

$$
\rho_{\text {loop }}^{*}\left(\Lambda_{g, n}\left(\gamma_{1}, \cdots, \gamma_{n}\right)\right)=\sum_{\alpha, \beta \in \mathscr{S}} \Lambda_{g-1, n+2}\left(\gamma_{1}, \cdots, \gamma_{n}, \alpha, \beta\right) \eta^{\alpha, \beta} .
$$

(iv) (Pairing)

$$
\int_{\overline{\mathcal{M}}_{0,3}} \Lambda_{0,3}\left(\mathbf{1}, \gamma_{1}, \gamma_{2}\right)=\eta\left(\gamma_{1}, \gamma_{2}\right)
$$

If in addition the following axiom holds

(v) (Flat identity) Let $\pi: \overline{\mathcal{M}}_{g, n+1} \rightarrow \overline{\mathcal{M}}_{g, n}$ be the forgetful morphism; then

$$
\Lambda_{g, n+1}\left(\gamma_{1}, \cdots, \gamma_{n}, \mathbf{1}\right)=\pi^{*} \Lambda_{g, n}\left(\gamma_{1}, \cdots, \gamma_{n}\right) .
$$

then we say that $\Lambda$ is a CohFT with a flat identity.

Note that $\Lambda_{0,3}$ will induce a Frobenius multiplication $\bullet$ on $(H, \eta)$, defined by

$$
\eta(\alpha \bullet \beta, \gamma)=\int_{\overline{\mathcal{M}}_{0,3}} \Lambda_{0,3}(\alpha, \beta, \gamma)
$$

We refer to $(H, \eta, \bullet)$ as the Frobenius algebra underlying $\Lambda$, or simply as the state space of $\Lambda$. The CohFT is called semisimple if the underlying Frobenius algebra is semisimple.

2.2. Examples of CohFTs. Let $\mathbb{C}^{N}$ be the complex vector space equipped with the standard bi-linear pairing: $\left(e_{i}, e_{j}\right)=\delta_{i, j}$. Let $\Delta=\left(\Delta_{1}, \cdots, \Delta_{N}\right)$ be a sequence of non-zero complex numbers. The following definition

$$
I_{g, n}^{N, \Delta}\left(e_{i_{1}}, \ldots, e_{i_{n}}\right):= \begin{cases}\Delta_{i}^{g-1+\frac{n}{2}} & \text { if } i=i_{1}=i_{2}=\cdots=i_{n}, \\ 0 & \text { otherwise }\end{cases}
$$

induces a CohFT on $\mathbb{C}^{N}$ which we call a rank $N$ trivial CohFT. The Frobenius algebra underlying $I^{N, \Delta}$ will be denoted by $\left(\mathbb{C}^{N}, \Delta\right)$. Note that the Frobenius multiplication is given by

$$
e_{i} \bullet e_{j}=\delta_{i j} \sqrt{\Delta_{i}} e_{i}
$$

Another famous example comes from Gromov-Witten theory (cf. [KM], CheR ). Let $X$ be a projective variety (or orbifold), let $H$ be its cohomology $H^{*}(X)$ (or Chen-Ruan cohomology $H_{\mathrm{CR}}^{*}(X)$ ), $\eta$ be the Poincaré pairing. Then $\Lambda_{g, n}^{X}(q)$ defined in (1.5) gives a CohFT for $q=0$. The above axioms make sense for cohomology classes $\Lambda_{g, n}^{X}(q)$ that have coefficients in some ring of formal power series. In such a case we say that we have a formal cohomological field theory. A priori, the CohFT in (1.5) is only formal. 
2.3. Givental's formalism. Following Givental, we introduce the vector space $\mathcal{H}=H((z))$ of formal Laurent series in $z^{-1}$. Furthermore, $\mathcal{H}$ is equipped with the following symplectic structure $\Omega$ :

$$
\Omega(f(z), g(z))=\operatorname{res}_{z=0}(f(-z), g(z)) d z, \quad f(z), g(z) \in \mathcal{H},
$$

where for brevity we put $(a, b)=\eta(a, b)$ for $a, b \in H$. Note that $\mathcal{H}$ has a polarization

$$
\mathcal{H}=\mathcal{H}_{+} \oplus \mathcal{H}_{-}
$$

with $\mathcal{H}_{+}=H[z]$ and $\mathcal{H}_{-}=z^{-1} H\left[\left[z^{-1}\right]\right]$, which allows us to identify $\mathcal{H} \cong$ $T^{*} \mathcal{H}_{+}$. We fix a Darboux coordinate system $q_{k}^{i}, p_{l, j}$ for $\mathcal{H}$ via

$$
f(z)=\sum_{k=0}^{\infty} \sum_{i=0}^{N-1} q_{k}^{i} \partial_{i} z^{k}+\sum_{l=0}^{\infty} \sum_{j=0}^{N-1} p_{l, j} \partial^{j}(-z)^{-l-1} \in \mathcal{H},
$$

For convenience, we put

$$
\mathbf{q}_{k}:=\left(q_{k}^{1}, \cdots, q_{k}^{N}\right) \quad \text { and } \quad \mathbf{q}:=\left(\mathbf{q}_{0}, \mathbf{q}_{1}, \cdots\right) .
$$

In this paper, we focus on the subgroup $\mathcal{L}^{(2)} \mathrm{GL}(H)$ of the loop group $\mathcal{L} \mathrm{GL}(H)$ consisting of symplectomorphisms $T: \mathcal{H} \rightarrow \mathcal{H}$. Note that such symplectomorphisms are defined by the following equation:

$$
{ }^{*} T(-z) T(z)=\mathrm{Id},
$$

where ${ }^{*} T$ is the adjoint operator with respect to the bi-linear pairing $\eta$, i.e.,

$$
\left({ }^{*} T f, g\right)=(f, T g) .
$$

We will allow symplectomorphism $E$ of the following form:

$$
E:=\operatorname{Id}+E_{1} z+E_{2} z^{2}+\cdots \in \operatorname{End}(H)[[z]] .
$$

They form a group which we denote by $\mathcal{L}_{+}^{(2)} \mathrm{GL}(H)$ and we refer to its elements as upper-triangular transformations.

Next, we want to define the quantization $\widehat{E}$. Note that $A=\log E$ is a welldefined infinitesimal symplectomorphism, i.e., ${ }^{*} A=-A$. For any infinitesimal symplectomorphism $A$, we can associate a quadratic Hamiltonian $h_{A}$ on $\mathcal{H}$,

$$
h_{A}(f)=\frac{1}{2} \Omega(A f, f) .
$$

The quadratic Hamiltonians are quantized by the rules:

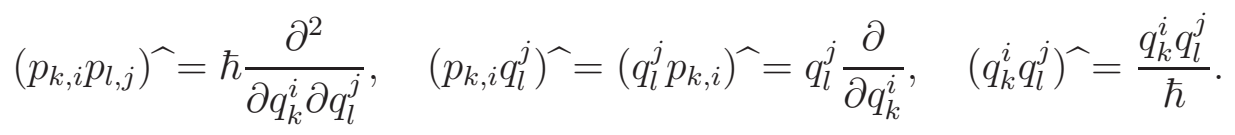

The quantization of $E$ is defined by

$$
\widehat{E}=e^{\widehat{A}}:=e^{\widehat{h_{A}}} \text {. }
$$


For an upper-triangular symplectomorphism $E$, there is an explicit formula for the quantization $\widehat{E}$. Put

$$
\mathbf{q}(z)=\sum_{k=0}^{\infty} \sum_{i=0}^{N-1} q_{k}^{i} \partial_{i} z^{k} \in H[[z]]
$$

Denote the dilaton shift by $\widetilde{\mathbf{q}}(z)=\mathbf{q}(z)+\mathbf{1} z$, i.e., $\widetilde{q}_{k}^{i}=q_{k}^{i}+\delta_{k}^{1} \delta_{0}^{i}$. Recall that the ancestor $G W$ potential of $X$ is

$$
\mathcal{A}^{X}(\hbar, \mathbf{q}(z)):=\exp \left(\sum_{g, n} \sum_{\beta \in \operatorname{NE}(X)} \sum_{\iota_{i}, k_{i}=0}^{\infty} \frac{\hbar^{g-1}\left\langle\tau_{\iota_{1}} \partial_{k_{1}}, \cdots, \tau_{\iota_{n}} \partial_{k_{n}}\right\rangle_{g, n, \beta}^{X} q^{\beta}}{n !} \prod_{i=1}^{n} \widetilde{q}_{k_{i}}^{i_{i}}\right) .
$$

$\mathcal{A}^{X}(\hbar, \mathbf{q}(z))$ belongs to a Fock space $\mathbb{C}\left[\left[\mathbf{q}_{0}, \widetilde{\mathbf{q}}_{1}, \mathbf{q}_{2}, \cdots\right]\right]$. The action of the quantization operator $\widehat{E}$, whenever it makes sense, is given by the following formula:

$$
\widehat{E}\left(\mathcal{A}^{X}(\hbar, \mathbf{q}(z))\right)=\left.\left(e^{W_{E}} \mathcal{A}^{X}(\hbar, \mathbf{q}(z))\right)\right|_{\mathbf{q} \mapsto E^{-1} \mathbf{q}},
$$

where $E^{-1} \mathbf{q}$ is the change of $\mathbf{q}$-coordinate

$$
\left(E^{-1} \mathbf{q}\right)_{k}^{i}=\sum_{l=0}^{k} \sum_{j=0}^{N-1}\left(E^{-1}\right)_{l}^{j i} q_{k-l}^{j} .
$$

And $W_{E}$ is the quadratic differential operator

$$
W_{E}:=\frac{\hbar}{2} \sum_{k, l=0}^{\infty} \sum_{i, j=0}^{N-1}\left(\partial^{i}, V_{k l}\left(\partial^{j}\right)\right) \frac{\partial^{2}}{\partial q_{k}^{i} \partial q_{l}^{j}},
$$

whose coefficients $V_{k l} \in \operatorname{End}(H)$ are given by

$$
\sum_{k, l \geq 0} V_{k l}(-z)^{k}(-w)^{l}=\frac{E^{*}(z) E(w)-\mathrm{Id}}{z+w} .
$$

Remark 2.1. Givental also considered the quantization of a general symplectomorphism of the form $e^{A}$. For example, A could be lower triangular in the sense containing the negative power of z. The lower triangular one can not be lift to cycle level. Hence, it will not be considered here.

2.4. Cycle-valued Quantization. Teleman [T] was able to lift the quantization of an upper triangular symplectic transformation to the level of cohomological field theory. Let us describe his construction. According to formula (2.7), the action of $\widehat{E}$ is a composition of two operations: exponential of the Laplace type operator (2.8) followed by the coordinate change $\mathbf{q} \mapsto E^{-1} \mathbf{q}$. 
2.4.1. Coordinate Change. Let $\Lambda_{g, n}$ be any multi-linear function on $H^{\otimes n}$ with values in the cohomology ring of $\overline{\mathcal{M}}_{g, n}$. We can extend $\Lambda_{g, n}$ from $H^{\otimes n}$ to $\mathcal{H}_{+}^{\otimes n}$ uniquely so that multiplication by $z$ is compatible with the multiplication by psi-classes, i.e.,

$$
\Lambda_{g, n}\left(\sum_{i \geq 0} \gamma_{1} z^{i}, \cdots\right)=\sum_{i \geq 0} \Lambda_{g, n}\left(\gamma_{1}, \cdots\right) \psi_{1}^{i} .
$$

Given an isomorphis of $\mathbb{C}[z]$-modules

$$
\Phi(z): H_{1}[[z]] \rightarrow H_{2}[[z]]
$$

we define

$$
(\Phi(z) \circ \Lambda)_{g, n}\left(\gamma_{1}, \cdots, \gamma_{n}\right)=\Lambda_{g, n}\left(\Phi(z)^{-1}\left(\gamma_{1}\right), \cdots, \Phi(z)^{-1}\left(\gamma_{n}\right)\right) \in H^{*}\left(\overline{\mathcal{M}}_{g, n}, \mathbb{C}\right) .
$$

Note that even if $\Lambda$ is a CohFT, $\Phi(z) \circ \Lambda$ might fail to be a CohFT.

2.4.2. Feynman type sum. The action of the exponential of the Laplacian (2.8) can be described in terms of sum over graphs. Let us explain this in some more details. For a given graph $\Gamma$ let us denote by $V(\Gamma)$ the set of vertices, $E(\Gamma)$ the set of edges, and by $T(V)$ the set of tails. For a fixed vertex $v \in V(\Gamma)$ we denote by $E_{v}(\Gamma)$ and $T_{v}(\Gamma)$ respectively the set of edges and tails incident with $v$. The graph is decorated in the following way: each vertex $v$ is assigned a non-negative number $g_{v}$ called genus of $v$; there is a bijection $t \mapsto m(t)$ between the set of tails and the set of integers $\{1,2, \ldots, \operatorname{Card}(T(\Gamma))\}$, and finally every flag $(v, e)$ (i.e., a pair consists a vertex and an incident edge) is decorated with a vector $z^{k} \partial^{i}(k \geq 0)$.

Furthermore, for a given edge $e$ we define a propagator $V_{e}$ as follows. Let $v^{\prime}$, $v^{\prime \prime}$ be the two vertexes incident with $e$ and let $z^{k^{\prime}} \partial^{i^{\prime}}$ and $z^{k^{\prime \prime}} \partial^{i^{\prime \prime}}$ be the labels respectively of the flags $\left(v^{\prime}, e\right)$ and $\left(v^{\prime \prime}, e\right)$; then we define

$$
V_{e}=\left(\partial^{i^{\prime}}, V_{k^{\prime}, k^{\prime \prime}} \partial^{i^{\prime \prime}}\right) \text {. }
$$

Note that since ${ }^{*} V_{k^{\prime}, k^{\prime \prime}}=V_{k^{\prime \prime}, k^{\prime}}$ the definition of $V_{e}$ is independent of the orientation of the edge $e$. For every vertex $v$ we define the differential operator

$$
D_{\mathbf{q}}^{v}=\prod_{e \in E_{v}(\Gamma)} \partial / \partial q_{k(e)}^{i(e)},
$$

where $z^{k(e)} \partial^{(i(e)}$ is the label of the flag $(v, e)$.

Given any formal function $\mathcal{A}(\hbar ; \mathbf{q})=\exp \left(\sum \hbar^{g-1} \mathcal{F}^{(g)}(\mathbf{q})\right)$ we have

$$
e^{W_{E}} \mathcal{A}(\hbar ; \mathbf{q})=\exp \left(\sum_{\Gamma} \frac{1}{|\operatorname{Aut}(\Gamma)|} \prod_{e \in E(\Gamma)} V_{e} \prod_{v \in V(\Gamma)} D_{\mathbf{q}}^{v} \mathcal{F}^{\left(g_{v}\right)}(\mathbf{q})\right),
$$

where the sum is over all connected decorated graphs $\Gamma$ and $|\operatorname{Aut}(\Gamma)|$ is the number of automorphisms of $\Gamma$ compatible with the decoration. 
Motivated by formula (2.11) we define

$$
\left(e^{W_{E}} \circ \Lambda\right)_{g, n}\left(\gamma_{1} \otimes \cdots \otimes \gamma_{n}\right)
$$

by the following formula:

$$
\sum_{\Gamma} \frac{1}{|\operatorname{Aut}(\Gamma)|} \prod_{e \in E(\Gamma)} V_{e} \prod_{v \in V(\Gamma)} \Lambda_{g_{v}, r_{v}+n_{v}}\left(\otimes_{e \in E_{v}(\Gamma)} \partial_{i(e)} \psi^{k(e)} \otimes_{t \in T_{v}(\Gamma)} \gamma_{m(t)}\right),
$$

where $r_{v}=\operatorname{Card}\left(E_{v}(\Gamma)\right), n_{v}=\operatorname{Card}\left(T_{v}(\Gamma)\right)$, and the sum is over all connected, decorated, genus-g graphs $\Gamma$ with $n$ tails. Note that this definition is compatible with (2.11) in a sense that the potential of the multi-linear maps (2.12) coincides with (2.11).

For an upper-triangular symplectic transformation $E$, we define

$$
\widehat{E} \circ \Lambda:=E \circ\left(e^{W_{E}} \circ \Lambda\right) .
$$

Using induction on the number of nodes, it is not hard to check that $\widehat{E} \circ \Lambda$ is a $\operatorname{CohFT}($ see $[\mathrm{T}]$ ).

2.4.3. Classification of semi-simple CohFT. Let $(H, \eta, \bullet)$ be a semi-simple Frobenius algebra. We pick an orthonormal basis $\left\{e_{i}\right\}$ of $H$, which allows us to identify $(H, \eta, \bullet)$ with the Frobenius algebra of a trivial CohFT, i.e.,, the state space of $I^{N, \Delta}$ for a particular $\Delta$ (see (2.2)). In this section we would like to recall the classification of all CohFTs whose state space is $(H, \eta, \bullet)$.

The space of such CohFTs admits the action (2.13) of the group $\mathcal{L}_{+}^{(2)} \mathrm{GL}(H)$. Note that this action does not change the Frobenius multiplication on $H$. On the other hand, the Abelian group $z^{2} H[z]$ (with group operation addition) acts on the space of CohFTs via translations. Namely, given $a(z) \in z^{2} H[z]$, we define

$$
\left(T_{a(z)} \circ \Lambda\right)_{g, n}\left(\gamma_{1}, \cdots, \gamma_{n}\right)
$$

by the fomula

$$
\sum_{k \geq 0} \frac{(-1)^{k}}{k !} \pi_{*}\left(\Lambda_{g, n+k}\left(\gamma_{1}, \cdots, \gamma_{n}, a(z), \cdots, a(z)\right)\right),
$$

where for each $k$, the map $\pi$ in the $k$-th summand is the map forgetting the last $k$ marked points. This action also preserves the Frobenius multiplication. Moreover, the following formula holds:

$$
T_{a(z)} \circ \widehat{E} \circ T_{a(z)}^{-1}=\widehat{E} \circ T_{a(z)-E^{-1} a(z)},
$$

i.e.,, we have an action of the group $z^{2} H[z] \rtimes \mathcal{L}_{+}^{(2)} \mathrm{GL}(H)$ on the set of CohFTs with state space $(H, \eta, \bullet)$. According to Teleman (see [T], Theorem 2) 
Theorem 2.2. ([T]) The orbit of the group $z^{2} H[z] \rtimes \mathcal{L}_{+}^{(2)} \mathrm{GL}(H)$ containing $I^{N, \Delta}$ consists of all CohFTs whose underlying Frobenius algebra is $(H, \eta, \bullet)$.

Let $a(z) \in z H[z]$ be arbitrary. Although the translation $T_{a(z)}$ is singular and will be not well defined after replacing multiplication by $z$ in terms of multiplication by psi-classes, the RHS of formula (2.15) always makes sense since $a(z)-E^{-1} a(z) \in z^{2} H[z]$. Therefore, we can define the following subgroup of $z^{2} H[z] \rtimes \mathcal{L}_{+}^{(2)} \mathrm{GL}(H)$ :

$$
\mathcal{L}_{a(z)}^{(2)} \mathrm{GL}(H)=T_{a(z)} \circ \mathcal{L}_{+}^{(2)} \mathrm{GL}(H) \circ T_{a(z)}^{-1} .
$$

The following fact follows easily from Theorem 2.2

Corollary 2.3. Let $a(z)=\mathbf{1} z$; then the orbit of the subgroup $\mathcal{L}_{a(z)}^{(2)} \mathrm{GL}(H)$ containing $I^{N, \Delta}$ consists of all CohFTs with a flat identity whose underlying Frobenius algebra is $(H, \eta, \bullet)$.

2.4.4. Higher-genus reconstruction. For $\mathbf{t}=\sum_{i=0}^{N-1} t_{i} \partial_{i} \in H$, we define a translation operator $T_{\mathbf{t}}$ acting on a CohFT $\Lambda$ by

$$
\left(T_{\mathbf{t}} \circ \Lambda\right)_{g, n}\left(\gamma_{1}, \cdots, \gamma_{n}\right):=\sum_{k \geq 0} \frac{(-1)^{k}}{k !} \pi_{*}\left(\Lambda_{g, n+k}\left(\gamma_{1}, \cdots, \gamma_{n}, \mathbf{t}, \cdots, \mathbf{t}\right)\right) .
$$

For brevity we put ${ }_{\mathbf{t}} \Lambda:=T_{\mathbf{t}} \circ \Lambda$. According to Teleman (see [T], Proposition 7.1), ${ }_{\mathbf{t}} \Lambda$ is a formal CohFT, i.e.,

$$
\left({ }_{\mathbf{t}} \Lambda\right)_{g, n} \in\left(H^{*}\left(\overline{\mathcal{M}}_{g, n}, \mathbb{C}\right) \otimes \mathbb{C}[[\mathbf{t}]]\right) \otimes\left(H^{\vee}\right)^{\otimes n}, \quad \mathbb{C}[[\mathbf{t}]]:=\mathbb{C}\left[\left[t_{0}, \cdots, t_{N-1}\right]\right] .
$$

It induces a ring structure on $H$ with the multiplication $\star_{\mathbf{t}}$ defined by

$$
\gamma_{1} \star_{\mathbf{t}} \gamma_{2}=\sum_{\alpha, \beta \in \mathscr{S}} \int_{\overline{\mathcal{M}}_{0,3}}\left({ }_{\mathbf{t}} \Lambda\right)_{0,3}\left(\gamma_{1}, \gamma_{2}, \alpha\right) \eta^{\alpha, \beta} \beta, \quad \gamma_{1}, \gamma_{2} \in H .
$$

Let us assume that the vector space $H$ is graded and that $\left\{\partial_{i}\right\}$ is a homogeneous basis with $\operatorname{deg}\left(\partial_{i}\right)=1-d_{i}$. We further assume that we are given an Euler vector field of the form

$$
\mathcal{E}=\sum_{i=0}^{N-1} d_{i} t_{i} \frac{\partial}{\partial t_{i}}+\sum_{j: d_{j}=0} r_{j} \partial_{j}
$$

where $r_{j}$ are some constants, so that the ring of formal power series $\mathbb{C}[[\mathbf{t}]]$ is a graded ring: an element $f(\mathbf{t})$ is homogeneous of degree $d_{f}$ iff $E(f(\mathbf{t}))=d_{f} f(\mathbf{t})$. The CohFT is called homogeneous of conformal weight $d$ if $H$ is graded, there exists an Euler vector field, and the maps:

$$
{ }_{\mathbf{t}} \Lambda_{g, n}: H^{\otimes n} \rightarrow H^{*}\left(\overline{\mathcal{M}}_{g, n} ; \mathbb{C}\right) \otimes \mathbb{C}[[\mathbf{t}]]
$$


are homogeneous of weight $d(g-1)+n$. Here the source of ${ }_{\mathbf{t}} \Lambda_{g, n}$ inherits the grading from $H$, the first tensor factor in the target is graded by halfing the degree of a cohomology class, and $\mathbb{C}[[\mathbf{t}]]$ is graded by $E$.

Let us assume further that the CohFT $\Lambda$ is generically semisimple, i.e.,, the ring structure $\star_{\mathbf{t}}$ is semisimple for generic $\mathbf{t}$. We denote by $u_{i}(\mathbf{t})$ the corresponding canonical coordinates, so that the map

$$
\Psi(\mathbf{t}): \mathbb{C}^{N} \rightarrow H, \quad e_{i} \mapsto \sqrt{\Delta_{i}(\mathbf{t})} \partial / \partial u_{i}(\mathbf{t})
$$

identifies the Frobenius algebras $\left(H, \eta, \boldsymbol{\star}_{\mathbf{t}}\right)$ and $\left(\mathbb{C}^{N}, \Delta(\mathbf{t})\right)$, where

$$
\Delta(\mathbf{t}):=\left(\Delta_{1}(\mathbf{t}), \ldots, \Delta_{N}(\mathbf{t})\right) .
$$

Let $U(\mathbf{t})$ be the diagonal matrix with entries $u_{i}(\mathbf{t}), 0 \leq i \leq N-1$. Following Givental we define an upper-triangular symplectic transformation $R(\mathbf{t})$, such that the formal asymptotical series $\Psi(\mathbf{t}) R(\mathbf{t}) e^{U(\mathbf{t})}$ is a solution to the differential equations (3.5) and (3.6). In fact, these equations determine $R(\mathbf{t})$ uniquely in terms of $\Psi(\mathbf{t})$ and $U(\mathbf{t})$ (see G2]). According to Teleman (see [T], Theorem 1) we have the following higher-genus reconstruction result.

Theorem 2.4. ([T]) If $\Lambda$ is a homogeneous CohFT with flat identity and $\star_{\mathbf{t}}$ is (formal) semi-simple; then

$$
{ }_{\mathbf{t}} \Lambda=\widehat{\Psi}(\mathbf{t}) \circ\left(T_{z} \circ \widehat{R}(\mathbf{t}) \circ T_{z}^{-1}\right) \circ I^{N, \Delta(\mathbf{t})},
$$

where $T_{z}:=T_{1}$.

Let us finish this section by drawing an important corrolary from the above theorem. The ancestor potential of a (singular) CohFT $\Lambda$ is a function of $a(z)=\sum_{i \geq 0} a_{i} z^{i} \in H[[z]]$, defined by

$$
\mathcal{A}(\Lambda, \hbar, a(z))=\exp \left(\sum_{g, n} \frac{\hbar^{g-1}}{n !} \int_{\overline{\mathcal{M}}_{g, n}} \Lambda_{g, n}(a(z), \cdots, a(z))\right) .
$$

Note that the translation $T_{z}^{-1}$ induces the so called dilaton shift, i.e, $\mathbf{q}(z) \mapsto$ $\widetilde{\mathbf{q}}(z)=: \mathbf{q}(z)+\mathbf{1} z$

$$
\mathcal{A}\left(T_{z}^{-1} \circ \Lambda, \hbar, \mathbf{q}(z)\right)=\mathcal{A}(\Lambda, \hbar, \widetilde{\mathbf{q}}(z)) .
$$

Furthermore, following Givental, the formal ancestor potential

$$
\mathcal{A}^{\text {formal }}(\mathbf{t})(\hbar, \mathbf{q}(z))
$$

of (the germ of) the Frobenius structure $\left(H, \eta, \boldsymbol{\star}_{\mathbf{t}}\right)$ is defined by

$$
\widehat{\Psi}(\mathbf{t}) \widehat{R}(\mathbf{t}) e^{(U(\mathbf{t}) / z)^{\wedge}} \prod_{i=1}^{\mu} \mathcal{A}^{\mathrm{pt}}\left(\hbar \Delta_{i}(\mathbf{t}),{ }^{i} \widetilde{\mathbf{q}}(z) \sqrt{\Delta_{i}(\mathbf{t})}\right),
$$

where $\widehat{\Psi}$ means change of the variables $\mathbf{q}(z) \mapsto \Psi^{-1}(\mathbf{t}) \mathbf{q}(z)$ and $\mathcal{A}^{\mathrm{pt}}$ is the total ancestor potential of the CohFT $I^{N=1, \Delta=1}$. 
Corollary 2.5. Under the same assumption as in Theorem 2.4 the following formula holds:

$$
\mathcal{A}\left({ }_{\mathbf{t}} \Lambda, \hbar, \widetilde{\mathbf{q}}(z)\right)=\mathcal{A}^{\text {formal }}(\mathbf{t})(\hbar, \mathbf{q}(z)) .
$$

Finally, let us point out that if $\Lambda^{X}$ is the CohFT induced from the GromovWitten theory of $X$; then

$$
\mathcal{A}^{X}(\hbar, \mathbf{q}(z)):=\mathcal{A}\left(\Lambda^{X}, \hbar, \widetilde{\mathbf{q}}(z)\right)
$$

coincides with the so called total ancestor potential of $X$. In particular, $\mathcal{A}^{\mathrm{pt}}$ in formula (2.20) is the total ancestor potential of a point.

\section{Global Frobenius manifolds For simple Elliptic Singularities}

In this section, we review the construction of global Frobenius manifolds for simple elliptic singularities. We follow MR.

3.1. Saito theory. K. Saito's theory of primitive forms [S1 yields a certain flat structure on the space of miniversal deformations of a singularity, which is known to be a Frobenius structure cf. [He, ST]. We refer to it as the Saito's Frobenius manifold structure. Let us recall the general set up.

Recall (see [ArGV]) the action of the group of germs of holomorphic changes of the coordinates $\left(\mathbb{C}^{N}, 0\right) \rightarrow\left(\mathbb{C}^{N}, 0\right)$ on the space of all germs at 0 of holomorphic functions. Let $\mathbf{x}=\left(x_{1}, \cdots, x_{N}\right) \in \mathbb{C}^{N}$. Given a holomorphic germ $f(\mathbf{x})$ with an isolated critical point at $\mathbf{x}=0$, we say that the family of functions $F(\mathbf{s}, \mathbf{x})$ is a miniversal deformation of $f$ if it is transversal to the orbit of $f$. One way to construct a miniversal deformation is to choose a $\mathbb{C}$-linear basis $\left\{\phi_{i}(\mathbf{x})\right\}_{i=0}^{\mu-1}$ in the Jacobi algebra $\mathcal{O}_{\mathbb{C}^{N}, 0} /\left\langle\partial_{x_{1}} f, \cdots, \partial_{x_{N}} f\right\rangle$. Here $\mu$ is the rank of the Jacobi algebra as a vector space, also known as the Milnor number or the multiplicity of the critical point. Then the following family provides a miniversal deformation:

$$
F(\mathbf{s}, \mathbf{x})=f(\mathbf{x})+\sum_{i=0}^{\mu-1} s_{i} \phi_{i}(\mathbf{x}), \quad \mathbf{s}=\left(s_{0}, s_{1}, \ldots, s_{\mu-1}\right) \in \mathcal{S},
$$

where $\mathcal{S} \subset \mathbb{C}^{\mu}$ is a small ball around $0 \in \mathbb{C}^{\mu}$. The domain of the function $F(s$,$) is chosen uniformly for s \in \mathcal{S}$ to be a certain open naighbourhood of $0 \in \mathbb{C}^{N}$, such that its boundary satisfies certain transversality conditions (see $\mathrm{ArGV}]$ ). Slightly abusing the notation we write $\mathbb{C}^{N}$, but we really mean an appropriate chosen open neighborhood of 0 . This should not cause any confusion. Moreover, we will apply Saito's theory only to singularities for which this special domain does coincide with $\mathbb{C}^{N}$.

Put $X=\mathcal{S} \times \mathbb{C}^{N}$ and let $C \subset X$ be the critical set of $F$, that is the support of the sheaf

$$
\mathcal{O}_{C}:=\mathcal{O}_{X} /\left\langle\partial_{x_{0}} F, \cdots, \partial_{x_{N-1}} F\right\rangle
$$


We have the following maps:

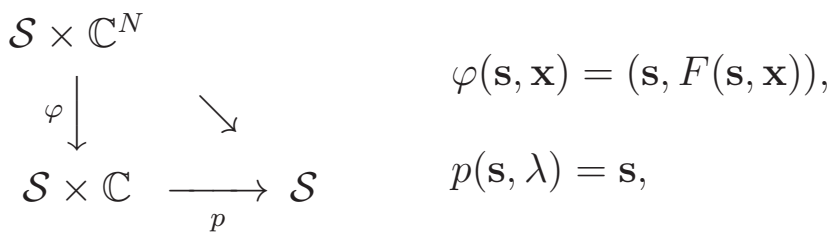

The map $\partial / \partial s_{i} \mapsto \partial F / \partial s_{i}$ induces an isomorphism between the sheaf $\mathcal{T}_{\mathcal{S}}$ of holomorphic vector fields on $\mathcal{S}$ and $q_{*} \mathcal{O}_{C}$, where $q=p \circ \varphi$. In particular, for any $\mathbf{s} \in \mathcal{S}$, the tangent space $T_{\mathbf{s}} \mathcal{S}$ is equipped with an associative commutative multiplication $\bullet_{\mathbf{s}}$ depending holomorphically on $\mathbf{s} \in \mathcal{S}$. In addition, if we have a volume form $\omega=g(\mathbf{s}, \mathbf{x}) d^{N} \mathbf{x}$, where $d^{N} \mathbf{x}=d x_{1} \wedge \cdots \wedge d x_{N}$ is the standard volume form; then $q_{*} \mathcal{O}_{C}$ (hence $\mathcal{T}_{\mathcal{S}}$ as well) is equipped with the residue pairing:

$$
\left\langle\psi_{1}, \psi_{2}\right\rangle=\frac{1}{(2 \pi i)^{N}} \int_{\Gamma_{\epsilon}} \frac{\psi_{1}(\mathbf{s}, \mathbf{y}) \psi_{2}(\mathbf{s}, \mathbf{y})}{F_{y_{1}} \cdots F_{y_{N}}} \omega,
$$

where $\mathbf{y}=\left(y_{1}, \cdots, y_{N}\right)$ are unimodular coordinates for the volume form, i.e., $\omega=d^{N} \mathbf{y}$, and $\Gamma_{\epsilon}$ is a real $N$-dimensional cycle supported on $\left|F_{x_{i}}\right|=\epsilon$ for $1 \leq i \leq N$.

Given a holomorphic function $f$ on $\mathbb{C}^{N}$ and a real number $m$ we define

$$
\operatorname{Re}_{f}^{m}\left(\mathbb{C}^{N}\right):=\left\{x \in \mathbb{C}^{N}: \operatorname{Re}(f(x)) \leq m\right\}
$$

Let

$$
J_{\mathcal{A}}(\mathbf{s}, z)=(-2 \pi z)^{-N / 2} z d_{\mathcal{S}} \int_{\mathcal{A}} e^{F(\mathbf{s}, \mathbf{x}) / z} \omega
$$

where $d_{\mathcal{S}}$ is the de Rham differential on $\mathcal{S}$ and $\mathcal{A}$ is a semi-infinite cycle from

$$
\lim _{\longleftarrow} H_{N}\left(\mathbb{C}^{N}, \operatorname{Re}_{F(\mathbf{s}, \cdot) / z}^{-m}\left(\mathbb{C}^{N}\right) ; \mathbb{C}\right) \cong \mathbb{C}^{\mu}
$$

By definition, the oscillatory integrals $J_{\mathcal{A}}$ are sections of the cotangent sheaf $\mathcal{T}_{\mathcal{S}}^{*}$. According to Saito's theory of primitive forms [S1], there exists a volume form $\omega$ such that the residue pairing is flat and the oscillatory integrals satisfy a system of differential equations, which in flat-homogeneous coordinates $\mathbf{t}=$ $\left(t_{0}, \ldots, t_{\mu-1}\right)$ have the form

$$
z \partial_{i} J_{\mathcal{A}}(\mathbf{t}, z)=\partial_{i} \bullet_{\mathbf{t}} J_{\mathcal{A}}(\mathbf{t}, z),
$$

where $\partial_{i}:=\partial / \partial t_{i}(0 \leq i \leq \mu-1)$ and the multiplication is defined by identifying vectors and covectors via the residue pairing. Due to homogeneity the integrals satisfy a differential equation with respect to the parameter $z \in$ $\mathbb{C}^{*}$ :

$$
\left(z \partial_{z}+\mathcal{E}\right) J_{\mathcal{A}}(\mathbf{t}, z)=\Theta J_{\mathcal{A}}(\mathbf{t}, z)
$$


where

$$
\mathcal{E}=\sum_{i=0}^{\mu-1} d_{i} t_{i} \partial_{i}, \quad\left(d_{i}:=\operatorname{deg} t_{i}=\operatorname{deg} s_{i}\right),
$$

is the Euler vector field and $\Theta$ is the so-called Hodge grading operator. The latter is defined by

$$
\Theta: \mathcal{T}_{S}^{*} \rightarrow \mathcal{T}_{S}^{*}, \quad \Theta\left(d t_{i}\right)=\left(1-\frac{D}{2}-d_{i}\right) d t_{i},
$$

where $D$ is the so called conformal dimension of the Frobenius manifold, uniquely determined by the symmetry of the degree spectrum: the numbers $d_{i}$ are symmetric with respect to the point $1-D / 2$. The compatibility of the system (3.5)-(3.6) implies that the residue pairing, the multiplication, and the Euler vector field give rise to a conformal Frobenius structure of conformal dimension $D$. We refer to $[\mathrm{D}, \mathrm{M}]$ for the definition and more details on Frobenius structures.

Theorem 3.1 ([He,, $\mathrm{ST}]$ ). Let $f$ be an isolated singularity, a primitive form $\omega$ induces a germ of Frobenius manifold structures $\left(T_{\mathbf{s}} \mathcal{S},\langle\rangle,, \bullet_{\mathbf{s}}, \mathcal{E}, \partial_{0}\right)$ with an Euler vector $\mathcal{E}$ and a flat identity $\partial_{0}$ for any $\mathbf{s} \in \mathcal{S}$. It is homogeneous and generically semisimple.

3.2. Global Frobenius manifold structures for simple elliptic singularities. Simple elliptic singularities are classified by K.Saito (cf. [S2]) into three different types, $\widetilde{E}_{6}, \widetilde{E}_{7}, \widetilde{E}_{8}$. In this paper, we consider three families of simple elliptic singularities by choosing a particular normal form in each family, see (3.7) below. The differential equations for the primitive forms will be the same under our choice, see (3.11). Besides, all the possible elliptic orbifolds $\mathbb{P}^{1}$ with three singular points can be seen as mirrors of our families at infinity of the complex plane, referred to as large complex structure limit point. The method also works for other normal forms although the mirrors of those elliptic orbifolds may appear in singular points on the complex plane other than the large complex structure limit points. For the framework of global mirror symmetry, see ChiR. Such global mirror symmetry phenomena for simple elliptic singularities are studied in [MS]. However, our choices here are enough for describing the quasi-modularity properties of CohFTs for those elliptic orbifolds. Only modular subgroups will be different for different normal forms. Let $W$ be one of the following three polynomials

$$
\widetilde{E}_{6}:=x_{1}^{3}+x_{2}^{3}+x_{3}^{3}, \quad \widetilde{E}_{7}:=x_{1}^{2} x_{3}+x_{1} x_{2}^{3}+x_{3}^{2}, \quad \widetilde{E}_{8}:=x_{1}^{3} x_{3}+x_{2}^{3}+x_{3}^{2} .
$$

Let us analyze the case $W=\widetilde{E}_{6}$. The other cases are similar. We define a 1-dimensional family by

$$
W_{\sigma}=W+\sigma x_{1} x_{2} x_{3}
$$


Note that $W_{\sigma}$ has an isolated singularity of the same rank $\mu$ iff $\sigma \in \Sigma$,

$$
\Sigma=\left\{\sigma \in \mathbb{C} \mid \sigma^{3}+27 \neq 0\right\} .
$$

Now we can replace $f$ in section 3.1 by $W_{\sigma}$. Its miniversal deformation is

$$
F:=W_{\sigma}(\mathbf{s}, \mathbf{x})=W_{\sigma}+\sum_{i=0}^{\mu-1} s_{i} \phi_{i} .
$$

Here $\left\{\phi_{i}\right\}_{i=0}^{\mu-1}$ is a basis of homogeneous polynomials of the Milnor ring $\mathscr{Q}_{W_{\sigma}}$. We always set $\phi_{\mu-1}=x_{1} x_{2} x_{3}, \phi_{0}=1$ and identify the index $\mu-1$ by -1 . Thus $\phi_{-1}=x_{1} x_{2} x_{3}$ and $s_{-1}=s_{\mu-1}$.

3.2.1. Primitive forms and global moduli of Frobenius manifolds. Recall that for a generic $(\mathbf{s}, \lambda)$, the fiber

$$
X_{\mathbf{s}, \lambda}=\left\{\mathbf{x} \in \mathbb{C}^{N} \mid \varphi(\mathbf{s}, \mathbf{x})=\lambda\right\}
$$

is homotopic to $\mu$ copies of $N-1$ dimensional sphere. The non-generic $(\mathbf{s}, \lambda)$ are the ones for which $X_{\mathbf{s}, \lambda}$ has a singularity. They form an analytic hypersurface called discirminant. The complement of the latter is a base for the middle cohomology bundle formed by the middle cohomology groups $H^{N-1}\left(X_{\mathbf{s}, \lambda} ; \mathbb{C}\right)$. In addition the integral structure in cohomology induces a flat Gauss-Manin connection.

Let us denote by $E_{\sigma}$ be the curve defined by $W_{\sigma}$,

$$
E_{\sigma}:=\left\{\left[x_{1}, x_{2}, x_{3}\right] \in \mathbb{C} P^{2} \mid W_{\sigma}\left(x_{1}, x_{2}, x_{3}\right)=0\right\} .
$$

One may compactify the family $X \rightarrow \mathcal{S} \times \mathbb{C}$ to $\bar{X} \rightarrow \mathcal{S} \times \mathbb{C}$ so that $E_{\sigma}=$ $\bar{X}-X$ is the boundary. $E_{\sigma}$ is also known as the elliptic curve at infinity, cf.[L]. According to K. Saito (see [S1]), the primitive forms for simple elliptic singularity $W_{\sigma}$ are homogenous of degree 0 and can be expressed as

$$
\omega=\frac{d^{3} \mathbf{x}}{\pi_{A}(\sigma)} .
$$

They are parametrized by the periods of $E_{\sigma}$,

$$
\pi_{A}(\sigma):=2 \pi i \int_{A_{\sigma}} \operatorname{Res}_{E_{\sigma}}\left[d^{3} \mathbf{x} / d F\right]
$$

where we fix a reference point $\sigma_{0} \in \Sigma, A \in H_{1}\left(E_{\sigma_{0}}, \mathbb{C}\right)$ is some fixed non-zero 1-cycle and $A_{\sigma}$ is a flat family of cycles uniquely determined by $A$ for all $\sigma$ in a small neighborhood of $\sigma_{0} \cdot d^{3} \mathbf{x} / d F$ is a holomorphic 2-form on $X_{\sigma, \lambda}$ and Res is the residue along $E_{\sigma}$. The boundary of any tubular neighborhood of $E_{\sigma}$ in 
$\bar{X}_{\mathrm{s}, \lambda}$ is a circle bundle over $E_{\sigma}$ that induces via pullback an injective tube map $L: H_{1}\left(E_{\sigma}\right) \rightarrow H_{2}\left(X_{\mathbf{s}, \lambda}\right)$. Let $\alpha=L(A)$; then we have

$$
\pi_{A}(\sigma)=\int_{\alpha} \frac{d^{3} \mathbf{x}}{d F}
$$

We refer to $\alpha$ as a tube or toroidal cycle. The space of all toroidal cycles coincides with the kernel of the intersection pairing on $H_{2}\left(X_{\mathbf{s}, \lambda} ; \mathbb{C}\right)$.

The space of all periods $\pi_{A}(\sigma)$ coincides with the space of solutions of the following differential equation (see the Appendix in [MR]),

$$
\frac{d^{2}}{d \sigma^{2}}+\frac{3 \sigma^{2}}{\sigma^{3}+27} \frac{d}{d \sigma}+\frac{\sigma}{\sigma^{3}+27}=0
$$

Take $\lambda=-\sigma^{3} / 27$, equation (3.11) is just a Gauss hypergeometric equation,

$$
\lambda(1-\lambda) \frac{d^{2}}{d \lambda^{2}}+\left(\frac{2}{3}-\frac{5 \lambda}{3}\right) \frac{d}{d \lambda}-\frac{1}{9}=0
$$

Now let us describe the global Frobenius manifold structure for those normal forms. We fix a symplectic basis $\left\{A^{\prime}, B^{\prime}\right\}$ of $H_{1}\left(E_{\sigma_{0}} ; \mathbb{Z}\right)$ once and for all. Then the primitive form is a multi-valued function on $\Sigma$. Thus it is more natural to replace $\Sigma$ by its universal cover. The latter is naturally identified with the upper half-plane $\mathbb{H}$. The points in the universal cover $\widetilde{\Sigma}$ of $\Sigma$ are pairs consisting of a point $\sigma \in \Sigma$ and a homotopy class of paths $l(s) \in \Sigma$ with $l(0)=\sigma_{0}, l(1)=\sigma$. The map

$$
(\sigma, l(s)) \mapsto \tau=\frac{\pi_{B^{\prime}}(\sigma)}{\pi_{A^{\prime}}(\sigma)}
$$

where the periods $\pi_{B^{\prime}}$ and $\pi_{A^{\prime}}$ are analytically continued along the path $l(s)$, defines an analytic isomorphism between the universal cover of $\widetilde{\Sigma}$ and the upper half-plane $\mathbb{H}$.

Let $\mathcal{M}=\mathbb{H} \times \mathbb{C}^{\mu-1}$. A global Frobenius structure exists on $\mathcal{M}$ for any non-zero cycle

$$
A=d A^{\prime}+c B^{\prime} \in H_{1}\left(E_{\sigma_{0}} ; \mathbb{C}\right), \quad-d / c \notin \mathbb{H} .
$$

Now let us describe the choice of a coordinate on $\mathbb{H}$, which we use through out the paper. Let $M$ be the classical monodromy operator on the middle homology bundle. By definition, $M$ is the linear operator induced by the parallel transport with respect to the Gauss-Manin connection along a loop in $\mathbb{C}^{*} \equiv\left\{\sigma_{0}\right\} \times(\mathbb{C} \backslash\{0\})$ based at $\lambda=1$. The operator $M$ is diagonalizable and one can find an eigenbasis $\left\{\alpha_{i}\right\}_{i=-1}^{\mu-2}, \alpha_{i} \in H^{*}\left(X_{\sigma, 1}, \mathbb{C}\right)$, s.t., the eigenvalue of $\alpha_{i}$ is $e^{2 \pi i d_{i}}$. Here

$$
(\sigma, 1):=(\sigma, 0, \cdots, 0,1) \in \mathcal{S} \times \mathbb{C}
$$


In particular, the invariant subspace of $M$ is spanned by $\alpha_{-1}$ and $\alpha_{0}$. Put $\alpha_{0}=-(-2 \pi)^{3 / 2} L(A)$ and $\alpha_{-1}=-(-2 \pi)^{3 / 2} L(B)$, where the cycle $B=b A^{\prime}+$ $a B^{\prime}$ is chosen to be any cycle linearly independent from $A$. Then it was proved in $\mathrm{MR}$, that the function

$$
t:=\frac{\pi_{B}(\sigma)}{\pi_{A}(\sigma)}=\frac{a \tau+b}{c \tau+d}
$$

is a flat coordinate. Slightly abusing the notation we simply write $t \in \mathbb{H}$ instead of saying that $t$ is given by formula (3.14) for some $\tau \in \mathbb{H}$. The entire flat coordinate system can be described in a similar way (see Section 2.2.2 in [MR]). Hence, $\mathcal{M}$ is a moduli space of global Frobenius manifold structures. For convenience, we denote the flat coordinates by $\mathbf{t}=\left(t, \mathbf{t}_{\geq 0}\right) \in \mathcal{M}$, with

$$
\mathbf{t}_{\geq 0}=\left(t_{0}, \cdots, t_{\mu-2}\right) \in \mathbb{C}^{\mu-1}
$$

3.3. The action of the monodromy group on flat coordinates. The monodromy group $\Gamma$ acts on $\mathcal{M}$ by covering transformations. In this subsection, we recall its action on flat coordinates.

Let $\nu$ be a monodromy transformation in the vanishing homology along a given loop $C$ in $\Sigma$ based at $\sigma_{0}$. According to [MR], the $\nu$ action on $\left\{\alpha_{i}\right\}_{i=-1}^{\mu-2}$ has a matrix form with respect to the vector of basis $\left(\alpha_{-1}, \cdots, \alpha_{\mu-2}\right)^{T}$,

$$
g \oplus \operatorname{Diag}\left(e^{2 \pi i d_{1} k}, \ldots, e^{2 \pi i d_{\mu-2} k}\right) \in \operatorname{SL}(2 ; \mathbb{C}) \times \mathbb{Z}^{\mu-2},
$$

where

$$
g\left(\alpha_{-1}\right)=n_{11} \alpha_{-1}+n_{12} \alpha_{0}, \quad \text { and } \quad g\left(\alpha_{0}\right)=n_{21} \alpha_{-1}+n_{22} \alpha_{0},
$$

and the matrix $\left(n_{i j}\right) \in \mathrm{SL}(2 ; \mathbb{C})$.

From now on we fix a flat coordinate system $t_{a}=t_{a}(\mathbf{s})(-1 \leq a \leq \mu-2)$, multi-valued on $\mathcal{S}$ and holomorphic on the cover $\mathcal{M}$, and denote by $H$ the space of flat vector fields on $\mathcal{M}$. We further assume that the flat coordinates are chosen in such a way that the residue pairing assumes the form:

$$
\left(\partial_{i}, \partial_{j}\right)=\delta_{i, j^{\prime}}, \quad-1 \leq i, j \leq \mu-2,
$$

where $\partial_{a}:=\partial / \partial t_{a}$ and ${ }^{\prime}$ is the involution defined by

$$
-1 \mapsto 0, \quad 0 \mapsto-1, \quad i \mapsto \mu-1-i, \quad 1 \leq i \leq \mu-2
$$

According to MR the flat coordinates can be expressed via certain period integrals as rational functions on the vanishing homology. It follows that the monodromy group $\Gamma$ acts on the flat coordinates as well and that this action coincides with the analytic continuation along $C$. According to $\mathrm{MR}$ if the flat coordinate system is such that the residue pairing has the above form; then 
the monodromy transformation (or equivalently the analytic continuation) of the flat coordinates has the following form. Put

$$
j_{\nu}(t):=j(g, t):=n_{21} t+n_{22} ;
$$

then

$$
\nu(\mathbf{t})_{-1}=g(t):=\frac{n_{11} t+n_{12}}{n_{21} t+n_{22}}
$$

and

$$
\nu(\mathbf{t})_{0}=t_{0}+\frac{n_{12}}{2 j_{\nu}(t)} \sum_{i=1}^{\mu-2} t_{i} t_{i^{\prime}}, \nu(\mathbf{t})_{i}=\frac{e^{2 \pi i d_{i} k}}{j_{\nu}(t)} t_{i}, \quad 1 \leq i \leq \mu-2 .
$$

\section{Global B-model CohFt and anti-holomorphic Completion}

The core of our paper is global B-model CohFTs for simple elliptic singularities, which we will construct in this section. The basic idea is that the global higher genus B-model theory of [MR can be enhanced to a global B-model CohFT using the construction of Teleman (see section two). The modularity will follow essentially from the monodromy calculations in [MR].

\subsection{Global B-model CohFT.}

4.1.1. Givental's semisimple quantization operator. Suppose that $W$ is one of the three families of simple elliptic singularities under consideration. Recall the global Frobenius manifold structures on $\mathcal{M}$. First we recall the definiton of Givental's quantization operator and then we use it to define a $\operatorname{CohFT} \Lambda^{W}(\mathbf{t})$ over the semisimple loci $\mathcal{M}_{s s}$.

Let $\mathcal{K} \subset \mathcal{M}$ be the set of points $\mathbf{t}$ such that $u_{i}(\mathbf{s}(\mathbf{t}))=u_{j}(\mathbf{s}(\mathbf{t}))$ for some $i \neq j$. We call this set the caustic and put $\mathcal{M}_{s s}$ for its complement. Note that the points $\mathbf{t} \in \mathcal{M}_{s s}$ are semisimple, i.e., the critical values $u_{i}(\mathbf{s}(\mathbf{t}))(1 \leq i \leq \mu)$ form a coordinate system locally near $\mathbf{t}$. Let $\mathbf{t} \in \mathcal{M}_{s s}$; then we have an isomorphism

$$
\Psi(\mathbf{t}): \mathbb{C}^{\mu} \rightarrow T_{\mathbf{t}} \mathcal{M}, \quad e_{i} \mapsto \sqrt{\Delta_{i}(\mathbf{s}(\mathbf{t}))} \frac{\partial}{\partial u_{i}(\mathbf{s}(\mathbf{t}))},
$$

where $\Delta_{i}(\mathbf{s}(\mathbf{t}))$ is defined by

$$
\left(\frac{\partial}{\partial u_{i}(\mathbf{s}(\mathbf{t}))}, \frac{\partial}{\partial u_{j}(\mathbf{s}(\mathbf{t}))}\right)=\frac{\delta_{i j}}{\Delta_{i}(\mathbf{s}(\mathbf{t}))}
$$

and we identify $T_{\mathbf{t}} \mathcal{M}$ with $H$ via the flat metric, i.e.,

$$
\frac{\partial}{\partial u_{i}}=\sum_{j=0}^{\mu-1} \frac{\partial t_{j}}{\partial u_{i}} \partial_{j}, \quad 1 \leq i \leq \mu
$$


$\Psi_{\mathbf{t}}$ diagonalizes the Frobenius multiplication and the residue pairing:

$$
e_{i} \bullet e_{j}=\delta_{i, j} \sqrt{\Delta_{i}(\mathbf{s}(\mathbf{t}))} e_{i}, \quad\left(e_{i}, e_{j}\right)=\delta_{i j} .
$$

The system of differential equations (3.5) and (3.6) admits a unique formal solution of the type

$$
\Psi(\mathbf{t}) R(\mathbf{t}) e^{U(\mathbf{t}) / z}, \quad R(\mathbf{t})=\operatorname{Id}+\sum_{k=1}^{\infty} R_{k}(\mathbf{t}) z^{k} \in \operatorname{End}\left(\mathbb{C}^{\mu}\right)[[z]] .
$$

where $U(\mathbf{t})$ is a diagonal matrix with entries $u_{1}(\mathbf{s}(\mathbf{t})), \ldots, u_{\mu}(\mathbf{s}(\mathbf{t}))$ on the diagonal, cf.[D, G1].

4.1.2. Global B-model CohFT. Givental used $R(\mathbf{t})$ to define a higher genus generating function over $\mathcal{M}_{s s}$. We would like to enhance his definition to Co$\mathrm{hFT}$. The main difficulty is to extend our definition to non-semisimple points in $\mathcal{K}$.

For any semisimple point $\mathbf{t} \in \mathcal{M}_{s s}$, we define a CohFT with a flat identity and a state space $H$ (see Sect. 2)

$$
\Lambda^{W}(\mathbf{t}):=\Psi(\mathbf{t}) \circ T_{z} \circ \widehat{R}(\mathbf{t}) \circ T_{z}^{-1} \circ I^{\mu, \Delta(\mathbf{t})} .
$$

We are interested in the loci of points $\mathbf{t}=(t, 0) \in \mathbb{H} \times \mathbb{C}^{\mu-1}$, which are never semisimple. To continue our B-model discussion, we need to prove that $\Lambda^{W}(\mathbf{t})$ extends holomorphically for all $\mathbf{t} \in \mathcal{M}$. To begin with, let us fix $g, n$, and $\gamma_{i} \in H$; for convenience, we denote by

$$
\Lambda_{g, n}^{W}(\mathbf{t}):=\left(\Lambda^{W}(\mathbf{t})\right)_{g, n} .
$$

$\Lambda_{g, n}^{W}(\mathbf{t})\left(\gamma_{1}, \ldots, \gamma_{n}\right)$ is a linear combination of cohomology classes on $\overline{\mathcal{M}}_{g, n}$ whose coefficients are functions on $\mathcal{M}$.

Lemma 4.1. The coefficients of $\Lambda_{g, n}^{W}(\mathbf{t})\left(\gamma_{1}, \ldots, \gamma_{n}\right)$ are meromorphic functions on $\mathcal{M}$ with at most finite order poles along the caustic $\mathcal{K}$.

Proof. By definition, the CohFT (4.1) depends only on the choice of a canonical coordinate system $u(\mathbf{t}):=\left(u_{1}\left(\mathbf{s}(\mathbf{t}), \ldots, u_{\mu}(\mathbf{s}(\mathbf{t}))\right.\right.$. The latter is uniquely determined up to permutation. Note that (4.1) is permutation-invariant, i.e., it does not matter how we order the canonical coordinates. On the other hand, up to a permutation $u(\mathbf{t})$ is invariant under the analytical continuation along a closed loop in $\mathcal{M}_{s s}$. It follows that $\Lambda_{g, n}^{W}(\mathbf{t})$ is a single valued function on $\mathcal{M}_{s s}$.

We need only to prove that the poles along $\mathcal{K}$ have finite order. Note that according to the definition of the class (2.12) only finitely many graphs $\Gamma$ contribute. The reason for this is that in order to have a non-zero contribution, we must have

$$
\sum_{e \in E_{v}(\Gamma)} k(e) \leq 3 g_{v}-3+r_{v}+n_{v}
$$


Summing up these inequalities, we get

$$
\sum_{v} \sum_{e \in E_{v}(\Gamma)} k(e) \leq 3(g-1)-3 \operatorname{Card}(E(\Gamma))+\sum_{v} r_{v}+n
$$

However

$$
\sum_{v} r_{v}=2 \operatorname{Card}(E(\Gamma))
$$

which implies that the number of edges of $\Gamma$ is bounded by $3 g-3+n$. This proves that there are finitely many possibilities for $\Gamma$. Moreover, there are only finitely many possibilities for $k(e)$, i.e., our class is a rational function on the entries of only finitely many $R_{k}$. Since each $R_{k}$ has only a finite order pole along the caustic the Lemma follows.

We will prove below that $\Lambda_{g, n}^{W}(\mathbf{t})$ is convergent near the point $(\sqrt{-1} \infty, 0) \in$ $\overline{\mathbb{H}} \times \mathbb{C}^{\mu-1}$ and that it extends holomorphically through the caustic (see Theorem 5.3 and Proposition 5.5). Thus $\Lambda^{W}(\mathbf{t})$ is a CohFT for all $\mathbf{t} \in \mathcal{M}$. In particular,

$$
\Lambda_{g, n}^{W}(t)=\lim _{\mathbf{t} \in \mathcal{M}_{s s} \rightarrow(t, 0)} \Lambda_{g, n}^{W}(\mathbf{t})
$$

for all $t \in \mathbb{H}=\mathbb{H} \times\{0\} \subset \mathcal{M}$.

4.2. Monodromy group action on $\Lambda_{g, n}^{W}(t)$. Using the residue pairing we identify $T^{*} \mathcal{M}$ and $T \mathcal{M}$, i.e., $d t_{i}=\partial_{i^{\prime}}$. We also identify $\operatorname{End}(H)$ with the space of $\mu \times \mu$ matrices via $A \mapsto\left(A_{i j}\right)$, where the entries $A_{i j}$ are defined in the standard way, i.e.,

$$
A\left(d t_{j}\right)=\sum_{i=-1}^{\mu-2} A_{i j} d t_{i} .
$$

Recall the notation from Section 3.3, a loop $C$ in $\Sigma$, inducing via the GaussManin connection a monodromy transformation $\nu$ on vanishing homology and a transformation of the flat coordinates via analytic continuation $\mathbf{t} \mapsto$ $\nu(\mathbf{t})$. The latter induces a monodromy transformation of the stationary phase asymptotics, which was computed in [MR, Lemma 4.1. In case $W=\widetilde{E}_{6}$, let

$$
M_{\nu}(\mathbf{t})=\left[\begin{array}{cccc}
j_{\nu}(t)^{-1} & * & * & * \\
0 & j_{\nu}(t) & 0 & 0 \\
0 & * & e^{4 \pi i k / 3} I_{3} & 0 \\
0 & * & 0 & e^{2 \pi i k / 3} I_{3}
\end{array}\right] \in \operatorname{End}(H)[[z]] .
$$

where

$$
M_{-1, j}=-e^{2 \pi i d_{j} k} n_{12} j_{\nu}^{-1}(t) t_{j}, \quad 1 \leq j \leq 6
$$

and

$$
M_{-1,0}=-n_{12} z-\frac{n_{12}^{2}}{2 j_{\nu}(t)} \sum_{i=1}^{6} t_{i} t_{i^{\prime}}, \quad M_{i, 0}=n_{12} t_{i^{\prime}}, \quad 1 \leq i \leq 6 .
$$


Lemma 4.2 ([MR ). The analytic continuation along $C$ transforms

$$
\Psi(\mathbf{t}) R(\mathbf{t}) e^{U(\mathbf{t}) / z} \quad \text { into } \quad{ }^{\mathrm{T}} M_{\nu}(\mathbf{t}) \Psi_{\mathbf{t}} R(\mathbf{t}) e^{U(\mathbf{t}) / z} P,
$$

where $P$ is a permutation matrix and ${ }^{\mathrm{T}}$ means transposition.

The CohFT constructed by the analytical continuation along $C$ of $\Lambda^{W}(\mathbf{t})$ will be denoted by

$$
\Lambda_{g, n}^{W}(\nu(\mathbf{t})) \in H^{*}\left(\overline{\mathcal{M}}_{g, n}, \mathbb{C}\right) \otimes\left(H^{\vee}\right)^{\otimes n} .
$$

Restricting to $\mathbf{t}_{\geq 0}=0$, we have

$$
{ }^{\mathrm{T}} M_{\nu}(t):=\lim _{\mathbf{t} \geq 0 \rightarrow 0}{ }^{\mathrm{T}} M_{\nu}(\mathbf{t})=j_{\nu}^{-1}(t) J_{\nu}(t) .
$$

With

$$
J_{\nu}(t):=\left[\begin{array}{cc}
1 & 0 \\
0 & j_{\nu}^{2}(t)
\end{array}\right] \oplus j_{\nu}(t) e^{4 \pi i k / 3} I_{3} \oplus j_{\nu}(t) e^{2 \pi i k / 3} I_{3} \in \operatorname{End}(H)[[z]] .
$$

Now let

$$
X_{\nu, t}(z)=\left[\begin{array}{cc}
1 & -n_{12} z / j_{\nu}(t) \\
0 & 1
\end{array}\right] \bigoplus I_{6} \in \operatorname{End}(H)[[z]] .
$$

Theorem 4.3. The analytic continuation transforms the Coh FT as follows:

$$
\Lambda^{W}(\nu(t))=J_{\nu}^{-1}(t) \circ \widehat{X}_{\nu, t}(z) \circ \Lambda^{W}(t) .
$$

Proof. The calculation in [MR] also works on cycle-valued level.

Now we give a lemma which is very useful later on.

Lemma 4.4. Let $E(z) \in \mathcal{L}_{+}^{(2)} \mathrm{GL}(H)$; then it intertwines with $J_{\nu}^{-1}(t)$ by

$$
J_{\nu}^{-1}(t) \circ \widehat{E}(z)=\widehat{E}\left(j_{\nu}^{2}(t) z\right) \circ J_{\nu}^{-1}(t) .
$$

Proof. From (4.4) and the definition of $J_{\nu}^{-1}(t) \circ$, we know that the pairing $\eta$ is scaled by $j_{\nu}^{2}(t)$ when applying $J_{\nu}^{-1}(t) \circ$. Thus the quadratic differential action $\widehat{E}(z)$ becomes $\widehat{E}\left(j_{\nu}^{2}(t) z\right)$.

4.3. Anti-holomorphic completion and modular transformation. Let $\mathscr{R}$ or $\mathcal{R}$ be a cohomology ring of any fixed Deligne-Mumford moduli space of stable curves of genus $g$ with $n$ marked points, i.e., $\mathscr{R}=H^{*}\left(\overline{\mathcal{M}}_{g, n}, \mathbb{C}\right)$ for some $2 g-2+n>0$.

Definition 4.5. We say that a $\mathscr{R}$-valued function $f: \mathbb{H} \rightarrow \mathscr{R}$ is a $\mathscr{R}$-valued quasi-modular form of weight $m$ with respect to some finite-index subgroup $\Gamma \subset \mathrm{SL}_{2}(\mathbb{Z})$ if there are $\mathscr{R}$-valued functions $f_{i}, 1 \leq i \leq K$, holomorphic on $\mathbb{H}$, such that

(i) The functions $f_{0}:=f$ and $f_{i}$ are holomorphic near cusp $\tau=i \infty$. 
(ii) The following $\mathscr{R}$-valued function

$$
f(\tau, \bar{\tau})=f_{0}(\tau)+f_{1}(\tau)(\tau-\bar{\tau})^{-1}+\cdots+f_{K}(\tau)(\tau-\bar{\tau})^{-K}
$$

is modular, i.e., there exists some $m \in \mathbb{N}$ such that for any $g \in \Gamma$,

$$
f(g \tau, g \bar{\tau})=j(g, \tau)^{m} f(\tau, \bar{\tau}) .
$$

$f(\tau, \bar{\tau})$ is called the anti-holomorphic completion of $f(\tau)$.

4.3.1. Anti-holomorphic completion of $\Lambda_{g, n}^{W}(t)$. Let $W$ be the homogeneous polynomial as in (3.7). Denote by

$$
X_{t, \bar{t}}(z)=\left[\begin{array}{cc}
1 & -z(t-\bar{t})^{-1} \\
0 & 1
\end{array}\right] \oplus I_{6} \in \operatorname{End}(H)[[z]],
$$

where $\bar{t}$ is the anti-holomorphic coordinate on $\mathbb{H}$ defined by (cf. formula (3.14))

$$
\bar{t}:=\frac{a \bar{\tau}+b}{c \bar{\tau}+d} .
$$

We define the anti-holomorphic completion of Coh FT $\Lambda^{W}(t)$ by:

$$
\Lambda^{W}(t, \bar{t}):=\widehat{X}_{t, \bar{t}}(z) \circ \Lambda^{W}(t) .
$$

Theorem 4.6. Under the assumption of extension property, the analytic continuation of the anti-holomorphic completion $\Lambda_{g, n}^{W}(t, \bar{t})$ along $\nu$ is

$$
J_{\nu}^{-1}(t) \circ \Lambda_{g, n}^{W}(t, \vec{t})
$$

Proof. We define an operator $\widehat{X}_{\nu, t, \bar{t}}(z)$, s.t., the following diagram is commutative:

$$
\begin{array}{cc}
\Lambda^{W}(t) \stackrel{\widehat{X}_{t, \bar{t}}(z)}{\longrightarrow} & \Lambda^{W}(t, \bar{t}) \\
\downarrow J_{\nu}(t) \circ \widehat{X}_{\nu, t}(z) & \\
\Lambda^{W}(\nu(t)) \stackrel{\widehat{X}_{\nu(t), \nu(\bar{t})}(z)}{\longrightarrow} & \Lambda^{W}(\nu(t), \nu(\bar{t}))
\end{array}
$$

We need to prove that

$$
\widehat{X}_{\nu, t, \bar{t}}(z)=J_{\nu}^{-1}(t)
$$

Let us consider the analytic continuation for $X_{t, \bar{t}}(z)$. Analytic continuation acts on $(t-\bar{t})^{-1}$ by

$$
\frac{1}{\nu(t)-\nu(\bar{t})}=-\left(\frac{n_{12}}{j_{\nu}(t)}+\frac{1}{t-\bar{t}}\right) j_{\nu}^{2}(t)
$$

By definition (4.7), this implies

$$
X_{\nu(t), \nu(\bar{t})}(z)=X_{t, \bar{t}}\left(j_{\nu}^{2}(t) z\right) X_{\nu, t}^{-1}\left(j_{\nu}^{2}(t) z\right) .
$$


Recalling Lemma 4.4, we get,

$$
J_{\nu}^{-1}(t) \circ \widehat{X}_{\nu, t}(z) \circ \widehat{X}_{t, \bar{t}}^{-1}(z)=\widehat{X}_{\nu, t}\left(j_{\nu}^{2}(t) z\right) \circ \widehat{X}_{t, \bar{t}}^{-1}\left(j_{\nu}^{2}(t) z\right) \circ J_{\nu}^{-1}(t) .
$$

Thus the result follows from (4.9) and (4.10).

4.3.2. Cycle-valued quasi-modular forms from $\Lambda_{g, n}^{W}(t)$. We consider a pair

$$
\left(\vec{\gamma}_{I}, \iota_{I}\right)=\left(\left(\gamma_{1}, \cdots, \gamma_{n}\right),\left(\iota_{1}, \cdots, \iota_{n}\right)\right) \in H^{\otimes n} \times \mathbb{Z}_{\geq 0}^{n}
$$

where each $\gamma_{i} \in \mathscr{S}=\left\{\partial_{-1}=\partial_{\mu-1}, \partial_{0}, \cdots, \partial_{\mu-2}\right\}$. I is a multi-index

$$
I=\left(i_{-1}, i_{0}, \cdots, i_{\mu-2}\right) \in \mathbb{Z}_{\geq 0}^{\mu}, \quad i_{-1}+\cdots+i_{\mu-2}=n .
$$

$i_{j}$ is the number of $i \in\{1, \cdots, n\}$ such that $\gamma_{i}=\partial_{j}$. Under the assumption of extension property, we define a cycle-valued function $f_{I, \iota_{I}}^{W}$ on $\mathbb{H}$,

$$
f_{I, l_{I}}^{W}(t)=\Lambda_{g, n}^{W}(t)\left(\vec{\gamma}_{I}\right) \in H^{*}\left(\overline{\mathcal{M}}_{g, n}, \mathbb{C}\right) .
$$

and its anti-holomorphic completion

$$
f_{I, \iota_{I}}^{W}(t, \bar{t}):=\Lambda_{g, n}^{W}(t, \bar{t})\left(\vec{\gamma}_{I}\right)
$$

For $\iota_{I}=(0, \cdots, 0)$, we simply denote them by $f_{I}^{W}(t)$ and $f_{I}^{W}(t, \bar{t})$. Let

$$
m(I):=2 i_{-1}+\sum_{j=1}^{\mu-2} i_{j} .
$$

Theorem 4.7. Let $W$ be a simple elliptic singularity. Then $f_{I, \iota_{I}}^{W}(t)$ satisfies the transformation law of cycle-valued quasi-modular forms of weight $m(I)$.

Proof. First we consider $\iota_{I}=(0, \cdots, 0)$. It is easy to see $f_{I}^{W}(t, \bar{t})$ is an antiholomorphic completion for $f_{I}^{W}(t)$ and for monodromy $\nu$ described as before, we have

$$
\begin{aligned}
f_{I}^{W}(\nu(t), \nu(\bar{t})) & =\left(\widehat{X}_{\nu, t, \bar{t}}(z) \circ \Lambda^{W}(t, \bar{t})\right)_{g, n}\left(\vec{\gamma}_{I}\right) \\
& =j_{\nu}^{m(I)}(t) \Lambda_{g, n}^{W}(t, \bar{t})\left(\vec{\gamma}_{I}\right) \\
& =j_{\nu}^{m(I)}(t) f_{I}^{W}(t, \bar{t}) .
\end{aligned}
$$

Now the statement follows from monodromy acts trivially on psi-classes.

Remark 4.8. For $f_{I}^{W}(t)$ to be a cycle-valued modular form, it needs to be holomophic at $\tau=\sqrt{-1} \infty$ (cf. formula (3.14) ). This will be achieved by the mirror theorem in section 5. Hence, by combining A-model with B-model, we produce cycle-valued quasi-modular forms. 


\section{A-Model CohFT and CyCle valued modular forms}

In the last section we constructed an anti-holomorphic modification of the B-model CohFT, such that it has the correct transformation property under analytic continuation. However, we still have to prove the following two properties: (1) the CohFT extends holomorphically through the caustic; (2) the quasi-modular forms are holomorphic at the cusp $\tau=\sqrt{-1} \infty$. We address both issues using an A-model (Gromov-Witten) CohFT and mirror symmetry. As a byproduct we obtain a geometric interpretation of the B-model CohFT as the Gromov-Witten CohFT of an elliptic orbifold $\mathbb{P}^{1}$ and we obtain a proof of our main result Theorem 1.2 .

The hard part of the argument is already completed in $\mathrm{KS}$, MR. Our goal is to recall the appropriate results and to show in what order they have to be used. The idea is as follows. We first establish analyticity and generic semisimplicity of the genus zero Gromov-Witten theory. This is done by using an estimate for the GW invariants and genus zero mirror symmetry. Then, we make use of a result of Coates-Iritani in order to prove the convergence of the Gromov-Witten ancestor CohFT of all genera. The last major step is a higher genus mirror symmetry that allows us to match the Gromov-Witten ancestor CohFT with the B-model CohFT near the large complex limit. This implies the extension property at $\tau=\sqrt{-1} \infty$. Finally, we use Lemma 3.2 from [MR] to conclude the extension property over entire B-model moduli space $\mathcal{M}$.

5.1. A-model. Let us recall a general mirror symmetry construction, called Berglund-Hübsch-Krawitz mirror symmetry. For a quasi-homogeneous polynomial $W$ with a suitable symmetry group $G$, a pair of mirror $\left(W^{T}, G^{T}\right)$ is constructed, [BH, $\left[\mathrm{K}\right.$. In our case, we choose a cubic polynomial $W^{T}$ with the maximal admissible group $G^{T}=G_{W^{T}}$, and consider this pair in A-model side. Its mirror will be the pair $(W, G=\{\operatorname{Id}\})$. So the B-model will be Saito-Givental's theory on the miniversal deformation of the family $W_{\sigma}$.

For $W=\widetilde{E}_{i}, i=6,7,8$ (see (3.7)) the mirror $W^{T}$ is given respectively by the following cubic polynomials:

$$
W^{T}=x_{1}^{3}+x_{2}^{3}+x_{3}^{3}, \quad x_{1}^{2} x_{2}+x_{2}^{3}+x_{1} x_{3}^{2}, \quad x_{1}^{3}+x_{2}^{3}+x_{1} x_{3}^{2} .
$$

The weights are $q_{i}=1 / 3$, for all $i=1,2,3$. Consider a hypersurface in the projective space,

$$
X_{W^{T}}=\left\{\left(x_{1}, x_{2}, x_{3}\right) \mid W^{T}\left(x_{1}, x_{2}, x_{3}\right)=0\right\} \hookrightarrow \mathbb{P}^{2} .
$$

Its maximal admissible group is

$$
G_{W^{T}}:=\left\{\left(\lambda_{1}, \lambda_{2}, \lambda_{3}\right) \in \mathbb{C}^{3} \mid W\left(\lambda_{1} x_{1}, \lambda_{2} x_{2}, \lambda_{3} x_{3}\right)=W^{T}\left(x_{1}, x_{2}, x_{3}\right)\right\} .
$$

It contains a subgroup $\langle J\rangle$, generated by the exponential grading element

$$
J:=\left(\exp \left(2 \pi i \cdot q_{1}\right), \exp \left(2 \pi i \cdot q_{2}\right), \exp \left(2 \pi i \cdot q_{3}\right)\right) \in G_{W^{T}} .
$$


$\langle J\rangle$ acts trivially on $X_{W^{T}}$. We denote by

$$
\widetilde{G}=G /\langle J\rangle .
$$

The quotient space

$$
\mathcal{X}_{W^{T}}:=X_{W^{T}} / \widetilde{G_{W^{T}}}
$$

is an elliptic orbifold with $\mathbb{P}^{1}$ as its underlying space. The A-model is the orbifold Gromov-Witten theory of $\mathcal{X}:=\mathcal{X}_{W^{T}}$.

5.2. Analyticity and generic semisimplicity. Let $H$ be the Chen-Ruan cohomology of $\mathcal{X}$ with unit $\mathbf{1}$ and Poincaré pairing $\eta$. Let the divisor $\mathcal{D}$ be a nef generator in $H^{2}(\mathcal{X}, \mathbb{Z}) \subset H_{\mathrm{CR}}^{2}(\mathcal{X}, \mathbb{Z})$ and let $\mathbf{t}=\left(t, t_{0}, t_{1} \ldots, t_{\mu-2}\right)$ be a linear coordinate system on $H$, such that $t$ is the coordinate along $\mathcal{D}$. Recall the Gromov-Witten $\operatorname{CohFT}_{\mathbf{t}} \Lambda^{\mathcal{X}}$, which a priori is only formal. Due to the so called divisor axiom we can identify $q=e^{t}$, i.e.,

$$
{ }_{\mathrm{t}} \Lambda_{g, n}^{\mathcal{X}}\left(\gamma_{1}, \cdots, \gamma_{n}\right) \in H^{*}\left(\overline{\mathcal{M}}_{g, n}, \mathbb{C}\right) \otimes \mathbb{C}\left[\left[e^{t}, t_{0}, \cdots, t_{\mu-2}\right]\right] .
$$

For every $\alpha, \beta, \gamma \in H$, the big quantum product $\alpha \star_{\mathbf{t}} \beta$ is defined by the relation

$$
\left\langle\alpha \star_{\mathbf{t}} \beta, \gamma\right\rangle={ }_{\mathbf{t}} \Lambda_{0,3}^{\mathcal{X}}(\alpha, \beta, \gamma) \text {. }
$$

The product is only formal in $\mathbf{t}$. We would like to prove that $\star_{\mathbf{t}}$ is convergent in the open polydisk $D_{\epsilon} \subset \mathbb{C}^{\mu}$ with center the origin and radius $\epsilon$, i.e, $(q=$ $\left.e^{t}, \mathbf{t}_{\geq 0}\right) \in D_{\epsilon}$. More precisely, our goal is to prove the following theorem.

Theorem 5.1. The following statements hold:

(1) There exists an $\epsilon>0$ such that ${ }_{\mathbf{t}} \Lambda_{0,3}^{\mathcal{X}}(\alpha, \beta, \gamma)$ is convergent for all $\left(q=e^{t}, \mathbf{t}_{\geq 0}\right) \in D_{\epsilon}$ and $\alpha, \beta, \gamma \in H$.

(2) The quantum product $\star_{\mathbf{t}}$ is generically semisimple.

Part (1) follows from Theorem 1.2 in [KS]. For the reader's convenience, we sketch the proof here as well. First let us denote by

$$
I_{0, n, d}^{G W}:=\max _{-1 \leq i_{j} \leq \mu-2}\left|\left\langle\partial_{i_{1}}, \cdots, \partial_{i_{n}}\right\rangle_{0, n, d}^{\mathcal{X}}\right| .
$$

By direct computation, $I_{0,3,0}^{G W} \leq 1$.

Lemma 5.2 (Lemma 4.16 in $[\mathrm{KS}])$. For $n+d \geq 4$, we have:

$$
I_{0, n, d}^{G W} \leq \begin{cases}d^{n-5} C^{n+d-4}, & d \geq 1 . \\ C^{n-4}, & d=0 .\end{cases}
$$

Here $C$ is some positive constant depending only on $n$. 
Since $H^{*}\left(\overline{\mathcal{M}}_{0,3}, \mathbb{C}\right) \cong \mathbb{C}$, it is enough to prove the convergence of the corresponding ancestor Gromov-Witten invariants. The divisor axiom implies

$$
\int_{\overline{\mathcal{M}}_{0,3}}{ }_{\mathbf{t}} \Lambda_{0,3}^{\mathcal{X}}(\alpha, \beta, \gamma)=\sum_{d \geq 0} q^{d} \sum_{k=0}^{\infty} \sum_{k_{0}+\cdots+k_{\mu-2}=k} \frac{\langle\alpha, \beta, \gamma, \cdots\rangle_{0,3+k, d}^{\mathcal{X}}}{k_{0} ! \cdots k_{\mu-2} !} \prod_{0 \leq i \leq \mu-2} t_{i}^{k_{i}}
$$

where the dots stand for the insertion $\partial_{0}, \cdots, \partial_{\mu-2}$ with multiplicities respectively $k_{0}, \ldots, k_{\mu-2}$. For dimensional reasons the Gromov-Witten invariants in the above formula vanish except for finitely many $k$. In another words,

$$
\int_{\overline{\mathcal{M}}_{0,3}}{ }_{\mathbf{t}} \Lambda_{0,3}^{\mathcal{X}}(\alpha, \beta, \gamma) \in \mathbb{C}\left[t_{0}, \cdots, t_{\mu-2}\right] \otimes \mathbb{C}[[q]] .
$$

Thus the convergence of ${ }_{\mathbf{t}} \Lambda_{0,3}^{\mathcal{X}}(\alpha, \beta, \gamma)$ in $\left(q, \mathbf{t}_{\geq 0}\right) \in D_{\epsilon}$ follows from the convergence of the following series near $q=0$,

$$
\sum_{d \geq 0} q^{d} d^{n-5} C^{n+d-4}
$$

Part (2) is not so easy to prove directly in the settings of Gromov-Witten theory. We use the genus-0 part of the mirror symmetry theorem of [KS]. We recall the genus-0 ancestor Gromov-Witten potential constructed from ${ }_{\mathbf{t}} \Lambda^{\mathcal{X}}$

$$
\mathcal{F}_{0}^{G W}(\mathcal{X})(\mathbf{t}):=\sum_{n} \sum_{\iota_{j}, i_{j}, d} \frac{1}{n !}\left\langle\tau_{\iota_{1}}\left(\partial_{i_{1}}\right), \cdots, \tau_{\iota_{n}}\left(\partial_{i_{n}}\right)\right\rangle_{0, n, d}^{\mathcal{X}}(\mathbf{t}) \prod_{j=1}^{n} \widetilde{q}_{i_{j}}^{\iota_{j}} .
$$

We can expand it as a formal series (due to the divisor axiom) as follows:

$$
\mathcal{F}_{0}^{G W}(\mathcal{X})(\mathbf{t}) \in \mathbb{C}\left[\left[q=e^{t}, t_{0}, \cdots, t_{\mu-2}\right]\right] \otimes \mathbb{C}\left[\left[\mathbf{q}_{0}, \widetilde{\mathbf{q}}_{1}, \mathbf{q}_{2}, \cdots\right]\right] .
$$

The space $\mathcal{M}=\mathbb{H} \times \mathbb{C}^{\mu-1}$ can be equipped with flat coordinates $\mathbf{t}^{B}$ corresponding to a choice of a primitve form (for $W$ ). In fact, $\mathcal{M}$ has a generically semi-simple Frobenius structure, which allows us to define the Saito-Givental ancestor potentials $\mathcal{F}_{g}^{S G}(W)\left(\mathbf{t}^{B}\right)$ for all genera $g$ (see 2.20 ).

The genus-0 mirror symmetry can be stated as follows: the primitive form can be chosen in such a way that there exists an analytic embedding $D_{\epsilon} \hookrightarrow \mathcal{M}$, called a mirror map, s.t.,

(1) The linear coordinates $\mathbf{t}$ on $D_{\epsilon}$ correspond to flat coordinates $\mathbf{t}^{B}$ on $\mathcal{M}$.

(2) We have

$$
\mathcal{F}_{0}^{G W}\left(\mathcal{X}_{W^{T}}\right)(\mathbf{t})=\mathcal{F}_{0}^{S G}(W)\left(\mathbf{t}^{B}\right)
$$

We denote the image of $D_{\epsilon}$ by $D_{\epsilon}^{B}$. Let us recall (see [MR]) that under the mirror map the modulus $\tau$ (cf. Sect. 3.2.1) is a flat coordinate on $\mathcal{M}$ and we have

$$
t=\frac{2 \pi \sqrt{-1}}{N} \tau
$$


where $N=3,4$, and 6 respectively for $W=\widetilde{E}_{6}, \widetilde{E}_{7}$, and $\widetilde{E}_{8}$. It follows that the large volume limit point $e^{t}=0$, i.e., $t=-\infty$ corresponds to the large complex structure limit point $\tau=\sqrt{-1} \infty$.

The proof can be splitted into two parts: choice of a primitive form, s.t., (1) holds and prove that the ancestor potentials on both sides are uniquely determined from a finite set of correlators, which agree under the mirror map. The first step was done in [MR and the second one in [KS].

5.3. Convergence of $\Lambda_{g, n}^{\mathcal{X}}(\mathbf{t})$. We identify via the mirror map the flat coordinates $\mathbf{t}^{B}$ on $\mathcal{M}$ and the linear coordinates $\mathbf{t}$ on $D_{\epsilon}$. Recall the CohFT $\Lambda_{g, n}^{W}\left(\mathbf{t}^{B}\right)$ defined by formula (4.1) for all semisimple points $\mathbf{t}^{B}$.

Theorem 5.3. The CohFT $\Lambda_{g, n}^{W}\left(\mathbf{t}^{B}\right)$ extends holomorphically for all $\mathbf{t}^{B} \in D_{\epsilon}^{B}$, the ancestor Gromov-Witten CohFT ${ }_{\mathbf{t}} \Lambda^{\mathcal{X}}$ is convergent for all $\mathbf{t} \in D_{\epsilon}$, and we have

$$
{ }_{\mathbf{t}} \Lambda_{g, n}^{\mathcal{X}}=\Lambda_{g, n}^{W}\left(\mathbf{t}^{B}\right), \quad \forall t \in D_{\epsilon} .
$$

Proof. The Frobenius structure of the quantum cohomology is generically semisimple (cf. Theorem 5.1, (2)). In particular, if we think of the CohFT ${ }_{\mathbf{t}} \Lambda^{\mathcal{X}}$ as a CohFT over the field

$$
\overline{\text { Frac } \mathbb{C}\left[\left[e^{t}, t_{0}, \cdots, t_{\mu-2}\right]\right]},
$$

where overline means algebraic closure and Frac stands for the field of fractions; then ${ }_{\mathbf{t}} \Lambda^{\mathcal{X}}$ is a semi-simple CohFT with a flat identity. Teleman's reconstruction Theorem 2.4 applies and we get that

$$
{ }_{\mathbf{t}} \Lambda_{g, n}^{\mathcal{X}}=\Lambda_{g, n}^{W}\left(\mathbf{t}^{B}\right)
$$

where the equality should be interpreted as equality in the space

$$
H^{*}\left(\overline{\mathcal{M}}_{g, n}, \mathbb{C}\right) \otimes \overline{\operatorname{Frac} \mathbb{C}\left[\left[e^{t}, t_{0}, \cdots, t_{\mu-2}\right]\right]} .
$$

On the other hand, according to Lemma (4.1), $\Lambda_{g, n}^{W}\left(\mathbf{t}^{B}\right)$ is meromorphic for $\mathbf{t} \in D_{\epsilon}^{B}$, thus

$$
\Lambda_{g, n}^{W}\left(\mathbf{t}^{B}\right)={ }_{\mathbf{t}} \Lambda_{g, n}^{\mathcal{X}} \in H^{*}\left(\overline{\mathcal{M}}_{g, n}, \mathbb{C}\right) \otimes \overline{\operatorname{Frac} \mathbb{C}\left\{e^{t}, t_{0}, \cdots, t_{\mu-2}\right\}},
$$

where $\mathbb{C}\left\{x_{1}, \ldots, x_{n}\right\}$ is the ring of convergent power series at $x_{1}=\cdots=x_{n}=0$ (the overline means algebraic closure). On the other hand, by definition

$$
{ }_{\mathbf{t}} \Lambda_{g, n}^{\mathcal{X}} \in H^{*}\left(\overline{\mathcal{M}}_{g, n}, \mathbb{C}\right) \otimes \mathbb{C}\left[\left[e^{t}, t_{0}, \cdots, t_{\mu-2}\right]\right] .
$$

Now we apply the following lemma of Coates-Iritani,

Lemma 5.4 ([CI1, Lemma 6.6). The intersection

$$
\overline{\text { Frac } \mathbb{C}\left\{x_{1}, \cdots, x_{n}\right\}} \cap \mathbb{C}\left[\left[x_{1}, \cdots, x_{n}\right]\right] \subset \overline{\text { Frac } \mathbb{C}\left[\left[x_{1}, \cdots, x_{n}\right]\right]}
$$

coincides with $\mathbb{C}\left\{x_{1}, \cdots, x_{n}\right\}$. 
This completes the proof.

5.4. Extension property. In this subsection, we use Lemma 3.2 from MR to derive the extension property.

Proposition 5.5. The coefficients of $\Lambda_{g, n}^{W}\left(\mathbf{t}^{B}\right)\left(\gamma_{1}, \ldots, \gamma_{n}\right)$ extend holomorphically through $\mathcal{K}$, i.e., they are holomorphic functions on $\mathcal{M}$.

Proof. Let us define an action of $\mathbb{C}^{*}$ on $\mathcal{M}=\mathbb{H} \times \mathbb{C}^{\mu-1}$ according to the weights of the coordinates $\mathbf{t}^{B}$. Since $\Lambda^{W}\left(\mathbf{t}^{B}\right)$ is a homogeneous CohFT, the domain $\widetilde{\mathcal{K}}$ of all $\mathbf{t}^{B}$ where the theory does not extend analytically is $\mathbb{C}^{*}$-invariant. Since $\widetilde{\mathcal{K}}$ is the set of points $\mathbf{t}^{B} \in \mathcal{M}$, such that $\Lambda^{W}\left(\mathbf{t}^{B}\right)$ has a pole, $\widetilde{\mathcal{K}}$ must be an analytic subset. Let us assume that $\widetilde{\mathcal{K}}$ is non-empty. The Hartogues extension theorem implies that the codimension of $\widetilde{\mathcal{K}}$ is at most 1 and hence precisely one. On the other hand, according to Theorem 5.3. the polydisk $D_{\epsilon}$ is disjoint from $\widetilde{\mathcal{K}}$. In particular, $\mathbb{H} \times\{0\}$ is not contained in $\widetilde{\mathcal{K}}$ and hence the two subvarieties interesect transversely. This combined with the $\mathbb{C}^{*}$ invariance of $\widetilde{\mathcal{K}}$ implies that the connected components of $\widetilde{\mathcal{K}}$ have the form $\left\{\tau_{0}\right\} \times \mathbb{C}^{\mu-1}$. This is a contradiction, because $\widetilde{\mathcal{K}} \subset \mathcal{K}$, while $\left\{\tau_{0}\right\} \times \mathbb{C}^{\mu-1} \not \subset \mathcal{K}$.

5.5. Quasi-modularity. Finally, let us complete the proof of our main theorem. According to Theorem 5.3 the Gromov-Witten CohFT $\Lambda_{g, n}^{\mathcal{X}}(q)$ is convergent and it coincides with $\Lambda_{g, n}^{W}(\tau)$, under the mirror map (5.4). The latter transforms as a quasi-modular form according to Theorem 4.7, it is analytic for all $\tau \in \mathbb{H}$ due to Proposition 5.5, and finally it extends holomorphically over the cusp $\tau=i \infty$ because $\Lambda_{g, n}^{\mathcal{X}}(q)$ extends holomorphically over $q=0$. This completes the proof of Theorem 1.2.

\section{REFERENCES}

[ABK] Aganagic, Mina; Bouchard, Vincent; Klemm, Albrecht. Topological strings and (almost) modular forms. Comm. Math. Phys. 277 (2008), no. 3, 771-819.

[AbGV] Abramovich, Dan; Graber, Tom; Vistoli, Angelo. Gromov-Witten theory of Deligne-Mumford stacks. Amer. J. Math. 130 (2008), no. 5, 1337-1398.

[ArGV] Arnol'd, V. I.; Gusein-Zade, S. M.; Varchenko, A. N. Singularities of differentiable maps. Vol. II. Monodromy and asymptotics of integrals. Monographs in Mathematics, 83. Birkhäuser Boston, Inc., Boston, MA, 1988. viii+492 pp.

[BCOV] M. Bershadsky, S. Cecotti, H. Ooguri, and C. Vafa, Kodaira-Spencer theory of gravity and exact results for quantum string amplitudes, Commun. Math. Phys. 165 (1994) 311-428

[BH] Berglund, Per; Hübsch, Tristan. A generalized construction of mirror manifolds. Nuclear Phys. B 393 (1993), no. 1-2, 377-391

[CKS] Chen, Yujun; Kontsevich, Maxim; Schwarz, Albert. Symmetries of WDVV equations. Nuclear Phys. B 730 (2005), no. 3, 352-363.

[CI1] Coates, Tom; Iritani, Hiroshi. On the Convergence of Gromov-Witten Potentials and Givental's Formula. arXiv:1203.4193v1 [math.AG] 
[CI2] Coates, Tom; Iritani, Hiroshi. In preparation.

[CheR] Chen, Weimin; Ruan, Yongbin. Orbifold Gromov-Witten theory. Orbifolds in mathematics and physics (Madison, WI, 2001), 25-85, Contemp. Math., 310, Amer. Math. Soc., Providence, RI, 2002.

[ChiR] Chiodo, Ruan. A global mirror symmetry framework for the LandauGinzburg/Calabi-Yau correspondence. Special Volume of the Ann. Inst. Fourier on the Workshop on Geometry and Physics of the Landau-Ginzburg model, Grenoble, $31 / 5-6 / 6 / 2010$.

[D] Dubrovin, Boris. Geometry of 2D topological field theories. Integrable systems and quantum groups (Montecatini Terme, 1993), 120-348, Lecture Notes in Math., 1620, Springer, Berlin, 1996.

[G1] Givental, Alexander B. Gromov-Witten invariants and quantization of quadratic Hamiltonians. Dedicated to the memory of I. G. Petrovskii on the occasion of his 100th anniversary, Mosc. J. 1 (2001), no. 4, 551-568, 645.

[G2] Givental, Alexander B. Semisimple Frobenius structures at higher genus. Internat. Math. Res. Notices 2001, no. 23, 1265-1286.

[He] Hertling, Claus. Frobenius manifolds and moduli spaces for singularities.

[HKQ] Huang, M.-x.; Klemm, A.; Quackenbush, S. Topological string theory on compact Calabi-Yau: modularity and boundary conditions. Homological mirror symmetry, 45-102, Lecture Notes in Phys., 757, Springer, Berlin, 2009.

[KM] Kontsevich Maxim. Gromov-Witten classes, quantum cohomology, and enumerative geometry. Comm. Math. Phys. 164(1994), no. 3, 525-562.

[K] Krawitz Marc. FJRW rings and Landau-Ginzburg Mirror Symmetry. arXiv:0906.0796v1 [math.AG]

[KS] Krawitz Marc; Shen Yefeng. Landau-Ginzburg/Calabi-Yau Correspondence of all Genera for Elliptic Orbifold $\mathbb{P}^{1}$. arXiv:1106.6270v1 [math.AG]

[L] Looijenga, Eduard. On the semi-universal deformation of a simple-elliptic hypersurface singularity. Unimodularity. Topology 16 (1977), no. 3, 257-262.

[LP] Lee, Y.-P.; Pandharipande, R. On semisimple Frobenius manifolds: I, II

[M] Manin, Yuri I. Frobenius manifolds, quantum cohomology, and moduli spaces. American Mathematical Society Colloquium Publications, 47. American Mathematical Society, Providence, RI, 1999. xiv+303 pp.

[MPT] Maulik, D.; Pandharipande, R.; Thomas, R. P. Curves on $K 3$ surfaces and modular forms. With an appendix by A. Pixton. J. Topol. 3 (2010), no. 4, 937-996.

[MR] Milanov Todor; Ruan Yongbin. Gromov-Witten theory of elliptic orbifold $\mathbb{P}^{1}$ and quasi-modular forms. arXiv:1106.2321v1 [math.AG]

[MS] Milanov Todor; Shen Yefeng. Global mirror symmetry for invertible simple elliptic singularities. In preparation.

[OP] Okounkov, A.; Pandharipande, R. Virasoro constraints for target curves. Invent. Math. 163 (2006), no. 1, 47-108.

[S1] Saito, Kyoji. On Periods of Primitive Integrals, I. Preprint RIMS(1982)

[S2] Saito, Kyoji. Einfach-elliptische Singularitäten. (German) Invent. Math. 23 (1974), 289-325.

[ST] Saito, Kyoji; Takahashi, Atsushi. From primitive forms to Frobenius manifolds. From Hodge theory to integrability and TQFT tt*-geometry, 31-48, Proc. Sympos. Pure Math., 78, Amer. Math. Soc., Providence, RI, 2008.

[T] Teleman, Constantin. The structure of 2D semisimple field theories. Invent. Math. Volume 188, Number 3 (2012), 525-588. 
[Z] Zhang Wei. Modularity of Generating Functions of Special Cycles on Shimura Varieties. PhD Thesis, Columbia University. 2009. 48 pp.

Kavli IPMU (WPI), The University of Tokyo, Kashiwa, Chiba 277-8583, JAPAN

E-mail address: todor.milanov@ipmu.jp

Department of Mathematics, University of Michigan, Ann Arbor, Mi 48105, USA

E-mail address: ruan@umich.edu

Department of Mathematics, University of Michigan, Ann Arbor, Mi 48105, USA

E-mail address: yf schen@umich.edu 